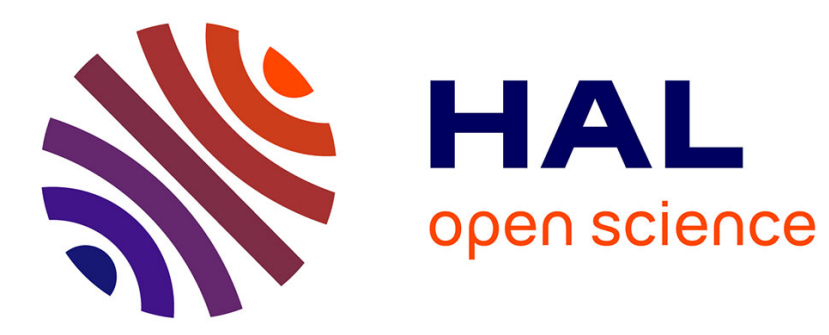

\title{
Homogenization of a spectral problem in neutronic multigroup diffusion
}

Grégoire Allaire, Yves Capdeboscq

\section{To cite this version:}

Grégoire Allaire, Yves Capdeboscq. Homogenization of a spectral problem in neutronic multigroup diffusion. Computer Methods in Applied Mechanics and Engineering, 2000, 187 (1-2), pp.91-117. 10.1016/S0045-7825(99)00112-7 . hal-02083805

\section{HAL Id: hal-02083805 https://hal.science/hal-02083805}

Submitted on 4 Aug 2020

HAL is a multi-disciplinary open access archive for the deposit and dissemination of scientific research documents, whether they are published or not. The documents may come from teaching and research institutions in France or abroad, or from public or private research centers.
L'archive ouverte pluridisciplinaire HAL, est destinée au dépôt et à la diffusion de documents scientifiques de niveau recherche, publiés ou non, émanant des établissements d'enseignement et de recherche français ou étrangers, des laboratoires publics ou privés. 


\title{
Homogenization of a spectral problem in neutronic multigroup diffusion
}

\author{
Grégoire Allaire* ${ }^{*} \quad$ Yves Capdeboscq $^{\dagger}$
}

December 2, 1998

\begin{abstract}
This paper is concerned with the homogenization of an eigenvalue problem in a periodic heterogeneous domain for the multigroup neutron diffusion system. Such a model is used for studying the criticality of nuclear reactor cores. We prove that the first eigenvector of the multigroup system in the periodicity cell controls the oscillatory behavior of the solutions, whereas the global trend is asymptotically given by a homogenized diffusion eigenvalue problem. The neutron flux, corresponding to the first eigenvector of the multigroup system, tends to the product of the first periodic and homogenized eigenvectors. This result justifies and improves the engineering procedure used in practice for nuclear reactor core computation.
\end{abstract}

\section{Introduction}

The power distribution in a nuclear reactor core is often obtained by solving an eigenvalue problem for a system of neutron diffusion equations. In a steadystate regime, such a system expresses the balance between neutrons produced by fission and neutrons absorbed or diffused by the medium. The unknown is a vector of neutron fluxes where each component corresponds to a given energy group, i.e. to neutrons with a given speed or kinetic energy. For a given bounded domain $\Omega$, this model reads

$$
\begin{cases}-\operatorname{div}(A(x) \nabla \varphi)+\Sigma(x) \varphi=\frac{1}{k_{e f f}} \sigma(x) \varphi & \text { in } \Omega, \\ \varphi=0 & \text { on } \partial \Omega\end{cases}
$$

where $A$ is the diffusion coefficient, $\Sigma$ the total cross section, $\sigma$ the fission cross section, and the Dirichlet boundary condition implies that no neutrons enter or leave the domain. In truth, the unknown is the couple $\left(k_{e f f}^{-1}, \varphi\right)$ of the first

* Laboratoire d'Analyse Numérique, Université Paris-VI, 75252 Paris Cedex 5, France, and CEA Saclay, DRN/DMT/SERMA, 91191 Gif-sur-Yvette, France (allaire@ann. jussieu.fr).

${ }^{\dagger} \mathrm{CEA}$ Saclay, DRN/DMT/SERMA, 91191 Gif sur Yvette, France (yves.capdeboscq@ecea.fr). 
eigenvalue and eigenvector for (1). The eigenvalue $k_{\text {eff }}$ is a measure of the balance between production and removal of neutrons in a quasistatic limit. If $k_{e f f}<1$, too many neutrons are diffused or absorbed in the core compared to their production by fission : the nuclear chain reaction dies out, and the reactor is said to be sub-critical. If $k_{e f f}>1$, too many neutrons are created by fission, and the reactor is said to be super-critical. In such a case, absorbing media (the so-called control rods) should be added to control the reaction. Eventually, when $k_{\text {eff }}=1$, the reactor is said to be critical : a perfect balance between fission and absorption-diffusion is obtained. Remark that (1) gives the spatial distribution of the neutron flux (which in turn yields the total power) but not its intensity since an eigenvector is defined up to a multiplicative constant. In section 2 it is checked that, under suitable assumptions, the first eigenvector of (1) is simple and positive which means that (1) makes physical sense (a neutron flux, as a density function, should be positive).

The diffusion model (1) is routinely used in many industrial codes for studying and optimizing nuclear reactor cores. Unfortunately, such domains are very heterogeneous, composed of more than 40000 different fuel rods immersed in a moderator (usually water), not to mention control rods, grids, and so on. Since a fine mesh is required, the direct computation of the solution is therefore long and expensive. Engineering procedures have been set up to obtain quick approximations of solutions. It amounts to homogenize (1) according to the following rule. The exact flux $\varphi$ is decomposed in the product of two terms

$$
\varphi(x)=\psi(x) u(x)
$$

where $\psi$ is a rapidly varying flux computed in sub-domains seen as periodic cells, and $u$ is a slowly varying flux computed in the whole domain with homogeneous averaged coefficients. More precisely (see e.g. [10], [15], [27], [28]), the microscopic flux $\psi$ is computed in each sub-domain $\Omega_{p}$ (typically a fuel assembly) as the solution $\psi_{p}=\psi_{\Omega_{p}}$ of the so-called infinite medium equation

$$
\begin{cases}-\operatorname{div}\left(A(x) \nabla \psi_{p}\right)+\Sigma(x) \psi_{p}=\mu_{p} \sigma(x) \psi_{p} & \text { in } \Omega_{p} \\ \frac{\partial \psi_{p}}{\partial n}=0 & \text { on } \partial \Omega_{p} .\end{cases}
$$

Then, averaged coefficients are evaluated by using some kinds of physically heuristic formulas as, for example in the one-energy-group case (other choices may be found in the above references and [23]),

$$
\bar{A}_{p}=\frac{\int_{\Omega_{p}} \psi_{p}(x) d x}{\int_{\Omega_{p}} \frac{\psi_{p}(x)}{A(x)} d x} \bar{\Sigma}_{p}=\frac{\int_{\Omega_{p}} \Sigma(x) \psi_{p}(x) d x}{\int_{\Omega_{p}} \psi_{p}(x) d x} \bar{\sigma}_{p}=\frac{\int_{\Omega_{p}} \sigma(x) \psi_{p}(x) d x}{\int_{\Omega_{p}} \psi_{p}(x) d x}
$$

The macroscopic flux $u(x)$ is then computed as a solution of (1) with the averaged coefficients (2), which are constant on each subdomain $\Omega_{p}$. This homogenization procedure works fine in many practical numerical computations. Recently, there has been a renewed interest in finding precise homogenization 
formulas, since the usual ones are not completely satisfactory in very heterogeneous cores (for example when mixing UO2 and new MOX assemblies, see e.g. [23]). The goal of this paper is to deliver precise homogenization formulas and to mathematically justify this entire homogenization procedure.

Although the homogenization method (using asymptotic expansions) is well established in neutron transport since the pionneering work of Larsen [22], it is only recently that its mathematical justification has been rigorously obtained for criticality problems. Indeed Malige [4], [23], [24] proved a complete convergence theorem for the homogenization of (1) in the one-energy-group case (the same problem was addressed by Dorning \& al. [16] using formal asymptotic expansions). Homogenization of criticality problems has also been rigorously justified in the context of neutron transport in [2], [3], [7], [8], [9].

In order to state precisely our main result, we introduce some notations. Let $\Omega$ be a bounded open set in $\mathbb{R}^{N}$ (the nuclear reactor core), and $Y=[0 ; 1]^{N}$ the unit periodicity cell (a typical fuel assembly). Let $\epsilon$ be a small positive parameter which is intended to tend to zero. The domain $\Omega$ is assumed to be periodic of period $\epsilon Y$. Since the period is decreasing, for physical reasons (namely, the mean free path of a neutron must stay of the order of the cell size) the diffusion is scaled to be of the order of $\epsilon^{2}$. Therefore, we shall study the homogenization of the following eigenvalue problem

$$
\begin{cases}-\epsilon^{2} \operatorname{div}\left(A\left(\frac{x}{\epsilon}\right) \nabla \phi^{\epsilon}\right)+\Sigma\left(\frac{x}{\epsilon}\right) \phi^{\epsilon}=\mu^{\epsilon} \sigma\left(\frac{x}{\epsilon}\right) \phi^{\epsilon} & \text { in } \Omega, \\ \phi^{\epsilon}=0 & \text { on } \partial \Omega,\end{cases}
$$

where $A(y), \Sigma(y)$ and $\sigma(y)$ are $Y$-periodic functions. Let $K$ denote the number of energy groups, i.e. the number of equations in the system (3). The unknown flux $\phi^{\epsilon}$ is a vector-valued function with $K$ components. The cross sections $\Sigma$ and $\sigma$ are $K \times K$ matrices, and the diffusion $A$ is a fourth-order tensor acting in the space of $K \times N$ matrices. We make a fundamental assumption about $A$ which is assumed to be a block-diagonal tensor, i.e. the components of system (3) are coupled only by zero-order terms. We emphasize that this assumption is physically not restrictive (see e.g. [12], [28]) and implies that the first eigenvector is positive as it should be since it is a density function. More details can be found in Section 2.

A particular case (and frequently used in practice) of (3) is the two-energygroup model $(K=2)$ which reads

$$
\begin{cases}-\epsilon^{2} \operatorname{div}\left(a_{1}\left(\frac{x}{\epsilon}\right) \nabla \phi_{1}^{\epsilon}\right)+\Sigma_{11}\left(\frac{x}{\epsilon}\right) \phi_{1}^{\epsilon} & =\mu^{\epsilon}\left(\sigma_{11}\left(\frac{x}{\epsilon}\right) \phi_{1}^{\epsilon}+\sigma_{12} \phi_{2}^{\epsilon}\left(\frac{x}{\epsilon}\right)\right) \\ -\epsilon^{2} \operatorname{div}\left(a_{2}\left(\frac{x}{\epsilon}\right) \nabla \phi_{2}^{\epsilon}\right)+\Sigma_{22}\left(\frac{x}{\epsilon}\right) \phi_{2}^{\epsilon} & =\Sigma_{21}\left(\frac{x}{\epsilon}\right) \phi_{1}^{\epsilon} \\ \phi_{1}^{\epsilon}=\phi_{2}^{\epsilon}=0 & \text { on } \partial \Omega\end{cases}
$$

where all coefficients are positive $Y$-periodic functions. The first component $\phi_{1}^{\epsilon}$ is the fast neutrons flux, and the second one $\phi_{2}^{\epsilon}$ is the slow (or thermal) neutrons flux. System (4) can be physically interpreted as follows : only fast neutrons are created by fission, while slow neutrons are generated by the slackening of fast neutrons, but both groups contribute to the fission source term. 
Our main result is the following homogenization theorem that we state in a loose way in order to avoid technicalities (for a rigorous statement, see Theorem 3.2 below).

Theorem 1.1 Let $\mu^{\infty}$ be the smallest eigenvalue and $\psi(y)$ a matching normalized eigenvector for the cell problem

$$
\left\{\begin{array}{l}
-\operatorname{div}(A(y) \nabla \psi)+\Sigma(y) \psi=\mu^{\infty} \sigma(y) \psi \quad \text { in } Y, \\
y \rightarrow \psi(y) Y-\text { periodic }
\end{array}\right.
$$

Let $\mu^{\epsilon, m}$ be the $m^{\text {th }}$ eigenvalue of (3) and $\phi^{\epsilon, m}$ an associated normalized eigenvector. Then, under a mild symmetry assumption for the coefficients (see (18)),

$$
\phi^{\epsilon, m}(x)=u^{m}(x) \psi\left(\frac{x}{\epsilon}\right)+o(1) \quad \text { and } \quad \mu^{\epsilon, m}=\mu^{\infty}+\epsilon^{2} \nu^{m}+o\left(\epsilon^{2}\right)
$$

where $\nu^{m}$ is the $m^{\text {th }}$ eigenvalue of the following homogenized one-group diffusion equation and $u^{m}$ is an associated scalar eigenvector

$$
\begin{cases}-\operatorname{div}(\bar{D} \nabla u)=\nu \bar{\sigma} u & \text { in } \Omega, \\ u=0 & \text { on } \partial \Omega,\end{cases}
$$

where $\bar{D}$ is a constant positive definite $N \times N$ matrix, and $\bar{\sigma}$ is a strictly positive constant, depending only on the coefficients $A, \Sigma$ and $\sigma$ (their precise values may be found in Section 3).

This result justifies, in the case of a periodic medium, the aforementioned engineering procedure of flux factorization and averaging but it delivers new homogenized formulas (at least to our knowledge). Remark that the microscopic flux $\psi$ is still the solution of a multi-group diffusion problem, but the macroscopic flux $u$ is indeed a scalar flux, solution of a one-group diffusion equation. As already mentioned, in the one-group case $K=1$, Theorem 1.1 has first been proved by Malige and his co-workers [4], [23], [24]. He also obtained formally the correct result in the two-group case $K=2$ by using two-scale asymptotic expansions. Eventually, [23], [24] contain many numerical computations demonstrating the efficiency of such an homogenization rule.

The paper is organized as follows. In Section 2 we give detailed assumptions on the coefficients and we recall regularity and existence results for systems (3) and (4). Such regularity results are needed to justify the factorization in the product of two terms. Section 3 is devoted to a precise statement of Theorem 1.1 and to its proof upon admitting the homogenization results of Section 5. Section 4 delivers energy-type formulas and a priori estimates, which implies the existence of two-scale limits. Then, Section 5 focus on the homogenization of a simpler associated source problem. Here, we use the two-scale convergence introduced in [1], [26]. Finally, in Section 6 we obtain further corrector results. 


\section{Existence and regularity results}

The goal of this section is to give precise assumptions on the coefficients of the multi-group diffusion system, and to establish some results concerning the existence and the regularity of its eigenvalues and eigenvectors. Most of the following theorems are variations of known results, and their proof is skipped or merely sketched.

Recall that $N$ is the space dimension, and $K$ is the number of energy groups. We adopt the convention that latin indices $i, j$ belong to $\{1, \ldots, N\}$, i.e. refer to spatial coordinates, while greek indices $\alpha, \beta$ vary in $\{1, . ., K\}$, i.e. refer to the group label.

Throughout this paper we shall use the following assumptions without mentioning them again. The first one is concerned with the diffusion tensor $A$. Denoting by $\left(\phi_{\alpha}\right)_{1 \leq \alpha \leq K}$ the components of the vector-valued flux $\phi$, its gradient is the $K \times N$ matrix $\nabla \phi$ defined by its entries

$$
\nabla \phi=\left(\frac{\partial \phi_{\alpha}}{\partial x_{i}}\right)_{1 \leq \alpha \leq K, 1 \leq i \leq N} .
$$

The current $A \nabla \phi$ is also a $K \times N$ matrix (its divergence has to be taken line by line as usual). We assume that $A$ is block diagonal, and we write $A=$ $\operatorname{diag}\left(A_{1}, \ldots, A_{K}\right)$, in the sense that

$$
A \nabla \phi=\left(A_{1} \nabla \phi_{1}, \ldots, A_{K} \nabla \phi_{K}\right)^{T},
$$

where each $\left(A_{\alpha}\right)_{1 \leq \alpha \leq K}$ is a symmetric $N \times N$ matrix. Taking into account (7) the spectral problem (3) is rewritten, for each $1 \leq \alpha \leq K$,

$$
\begin{cases}-\epsilon^{2} \operatorname{div}\left(A_{\alpha}\left(\frac{x}{\epsilon}\right) \nabla \phi_{\alpha}^{\epsilon}\right)+\sum_{\beta=1}^{K} \Sigma_{\alpha, \beta}\left(\frac{x}{\epsilon}\right) \phi_{\beta}^{\epsilon}=\mu^{\epsilon} \sum_{\beta=1}^{K} \sigma_{\alpha, \beta}\left(\frac{x}{\epsilon}\right) \phi_{\beta}^{\epsilon} & \text { in } \Omega, \\ \phi_{\alpha}^{\epsilon}=0 & \text { on } \partial \Omega,\end{cases}
$$

which makes it a system of $K$ equations coupled only through zero-order terms. This is a classical assumption which is physically not restrictive (see e.g. [12], [28]).

Our second assumption is that all coefficients in (8) are measurable and bounded, i.e. $A_{\alpha, i j}(y), \Sigma_{\alpha, \beta}(y), \sigma_{\alpha, \beta}(y) \in L^{\infty}(Y)$ for $1 \leq i, j \leq N$ and $1 \leq$ $\alpha, \beta \leq K$. This is the natural functional framework since we want to model heterogeneous media having discontinuous properties. Furthermore, the diffusion matrices are assumed to be coercive, i.e. there exists a positive constant $C>0$ such that, for any $\alpha \in\{1, \ldots, K\}$ and for any $\xi \in \mathbb{R}^{N}$,

$$
A_{\alpha}(y) \xi \cdot \xi \geq C|\xi|^{2} \text { for a.e. } y \in Y \text {. }
$$

For physical reasons, all fission cross-sections are non-negative $\sigma_{\alpha, \beta} \geq 0$ (fission is a production process), while the matrix $\Sigma$ of the total (or scattering) crosssections is diagonal dominant, i.e. $\Sigma_{\alpha, \alpha} \geq 0, \Sigma_{\alpha, \beta} \leq 0$ if $\alpha \neq \beta$, and $\sum_{\beta=1}^{K} \Sigma_{\alpha, \beta} \geq$ 
0 (this means that there is a net absorption in each group). For mathematical reasons (mainly for Theorem 2.3 below), we need slightly stronger assumptions, namely that there exists a positive constant $C>0$ such that, a.e. in $Y$,

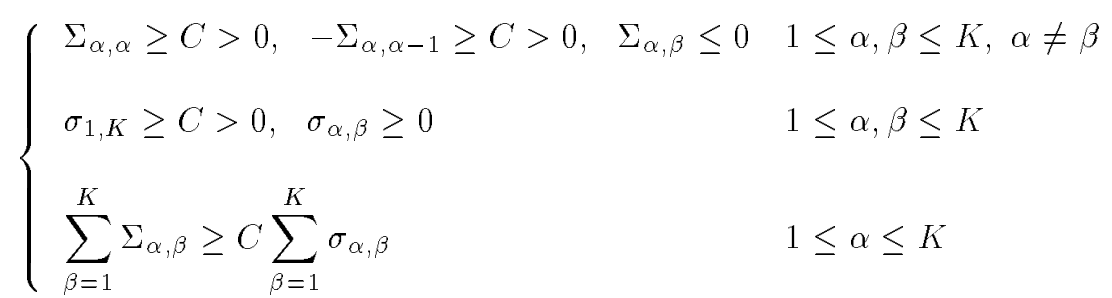

Finally, our third assumption is that the nuclear reactor core is periodic, i.e. all coefficients $A(y), \Sigma(y)$, and $\sigma(y)$ are $Y$-periodic functions. This hypothesis is crucial for the homogenization procedure. In particular our results do not hold true any longer if the coefficients are the product of periodic functions with macroscopic modulations, as for example $\Sigma\left(x, \frac{x}{\epsilon}\right)$ with a $Y$-periodic function $\Sigma(x, y)$. Let us mention however that some small perturbations of order $\epsilon^{2}$ of the cross sections can be allowed (see Remark 4.3).

Remark 2.1 The second line of (10) implies that fission occurs everywhere in the nuclear reactor core. This is not completely satisfactory since a core is a mixture of fissile materials and moderators where no fission occurs (for example, in pressurized water reactors, fission occurs in the fuel rods but not in the water surrounding the rods). However, as is shown in [28], if $Y_{0}$ is a non-empty open subset of $Y$, one can replace (10) by

$$
\left\{\begin{array}{ll}
\Sigma_{\alpha, \alpha} \geq C>0, \quad \Sigma_{\alpha, \beta} \leq 0 & 1 \leq \alpha, \beta \leq K, \alpha \neq \beta \\
\sigma_{\alpha, \beta} \geq 0 & 1 \leq \alpha, \beta \leq K \\
\sum_{\beta=1}^{K} \Sigma_{\alpha, \beta} \geq C \sum_{\beta=1}^{K} \sigma_{\alpha, \beta} & 1 \leq \alpha \leq K
\end{array} \quad \text { a.e. in } Y,\right.
$$

and

$$
\left\{\begin{array}{l}
-\Sigma_{\alpha, \alpha-1} \geq C>0 \quad 1 \leq \alpha \leq K \\
\sigma_{1, K} \geq C
\end{array} \quad \text { a.e. in } Y_{0},\right.
$$

where the only change is in (12) which holds only in $Y_{0}$. In [28] (11-12) is shown to yield the same results than (10) only for the two-group diffusion system, but it is clear that all results in this section hold true also with this weaker assumption for any $K \geq 2$.

In the one-energy group case $K=1$, since the diffusion matrix $A$ is symmetric, equation (3) defines a compact self-adjoint operator acting in $L^{2}(\Omega)$. Therefore, for any fixed $\epsilon>0$, a well-known result asserts the existence of solutions to (8) and its regularity. 
Theorem 2.2 Let the number of group be $K=1$. Under assumptions (7), (9), and (10), problem (8) has a countable number of real positive eigenvalues. The first (smallest) eigenvalue is simple and has an associated positive eigenfunction in $\Omega$. Furthermore, all eigenfunctions belong to $H_{0}^{1}(\Omega) \cap C^{0, s}(\Omega)$ for some $s>0$.

Theorem 2.2 is classical. The fact that the spectrum is a countable discrete set is due to the compactness of the operator. The regularity result may be found e.g. in [17]. The fact that the minimum eigenvalue is simple and has a positive eigenfunction is a consequence of the Krein-Rutman Theorem [21]. The same result holds also for the periodic problem (5).

The generalization of Theorem 2.2 to the multi-group case $K \geq 2$ is less obvious. In particular, system (8) is not self-adjoint. We first address the existence of solutions, and then turn to the regularity question.

Theorem 2.3 Under assumptions (7), (9), and (10), problem (8) admits at least one, and at most a countable number of eigenvalues (possibly complex) with associated eigenvectors in $H_{0}^{1}(\Omega)^{K}$. Furthermore, the first eigenvalue of (8) (i.e. the smallest in modulus) is real and simple, and its corresponding eigenvector can be chosen to be positive in $\Omega$ (i.e. each component is positive).

Remark 2.4 Throughout the paper, we label the eigenvalues by increasing order of their modulus, and we normalize the eigenvectors such that their $L^{2}$-norm is equal to 1 .

Theorem 2.3 has first been proved by Habetler and Martino [18], with the help of Green functions inequalities given by Stampacchia and of the KreinRutman Theorem (see [30], [21], [5]). A modern exposition of this result may be found in the book of Planchard [28]. In this later reference, Theorem 2.3 is shown to hold true also if assumption (10) is replaced by (11-12) which is weaker but more realistic (a complete proof is only given in the 2-energy-groups case).

The factorization principle described in the introduction is based on the following eigenvalue problem in the unit cell $Y$ (the so-called infinite medium equation)

$$
\left\{\begin{array}{l}
-\operatorname{div}(A(y) \nabla \psi)+\Sigma(y) \psi=\mu^{\infty} \sigma(y) \psi \\
y \rightarrow \psi(y) \text { Y-periodic }
\end{array}\right.
$$

where $\mu^{\infty}$ is the first eigenvalue. In order to compute the homogenized coefficients, we also need to introduce the adjoint cell problem of (13)

$$
\left\{\begin{array}{l}
-\operatorname{div}\left(A(y) \nabla \psi^{*}\right)+\Sigma^{*}(y) \psi^{*}=\mu^{\infty} \sigma^{*}(y) \psi^{*} \\
y \rightarrow \psi^{*}(y) \text { Y-periodic, }
\end{array}\right.
$$

where $\Sigma^{*}$ and $\sigma^{*}$ are the adjoint or transposed matrices of $\Sigma$ and $\sigma$ respectively, and $\mu^{\infty}$ is the first eigenvalue (the same as for (13)). Throughout this paper we denote by $\left(\psi_{\alpha}\right)_{1 \leq \alpha \leq K}$ (resp. $\left.\left(\psi_{\alpha}^{*}\right)_{1 \leq \alpha \leq K}\right)$ the components of the eigenvector $\psi$ of (13) (resp. $\psi^{*}$ of (14)) associated to the first eigenvalue $\mu^{\infty}$. Of course, for these two cell problems an obvious generalization of Theorem 2.3 holds. 
Corollary 2.5 Under assumptions (7), (9), and (10), the cell problems (13) and (14) admit at least one, and at most a countable number of eigenvalues with associated eigenvectors in $H_{\#}^{1}(Y)^{K}$. Furthermore, they have a common first eigenvalue $\mu^{\infty}$ which is real and simple, and its corresponding eigenvectors $\psi$ and $\psi^{*}$ can be chosen to be positive in $Y$.

We recall that $H_{\#}^{1}(Y)$ is the subspace of $H_{l o c}^{1}\left(\mathbb{R}^{N}\right)$ made of $Y$-periodic functions. We now turn to the regularity of the eigenfunctions. Since this extra smoothness is required in the sequel only for the first eigenfunctions of (13) and (14), we state this result only for these cell problems.

Proposition 2.6 The eigenfunctions of the cell problems (13) and (14) are Hölder continuous, i.e. belong to $\left[H_{\#}^{1}(Y) \cap C_{\#}^{0, s}(Y)\right]^{K}$ for some $s>0$.

The proof of Proposition 2.6 is based on regularity results due to Stampacchia [30] and a boot-strap argument (starting from $L^{2}(Y)$ the regularity of the right hand side is iteratively increased up to $L^{q}(Y)$ with $q>N / 2$ which implies that the solution is continuous). The argument is quite standard so we omit it. Of course, assumption ( 7 ) on the diagonal character of the diffusion tensor is crucial here.

\section{$3 \quad$ Main results}

This section is devoted to a presentation of our main results of homogenization. We begin by recalling the homogenization theorem proved by Malige [4], [23] in the one-group case $K=1$. It is simpler to state in this case, and its proof is both simple and enlighting (see below).

Theorem 3.1 Assume that the number of energy group is $K=1$. Let $\psi$ and $\mu^{\infty}$ be the first eigenvector and eigenvalue of the cell problem (13). For $m \geq 1$, let $\mu^{\epsilon, m}$ and $\phi^{\epsilon, m}$ be the $m^{\text {th }}$ eigenvalue and normalized eigenvector of (8). Then,

$$
\phi^{\epsilon, m}(x)=u^{\epsilon, m}(x) \psi\left(\frac{x}{\epsilon}\right) \quad \text { and } \quad \mu^{\epsilon, m}=\mu^{\infty}+\epsilon^{2} \nu^{m}+o\left(\epsilon^{2}\right),
$$

where, up to a subsequence, the sequence $u^{\epsilon, m}$ converges weakly in $H_{0}^{1}(\Omega)$ to $u^{m}$, and $\left(\nu^{m}, u^{m}\right)$ is the $m^{\text {th }}$ eigenvalue and eigenvector for the homogenized problem

$$
\begin{cases}-\operatorname{div}(\bar{D} \nabla u)=\nu \bar{\sigma} u & \text { in } \Omega, \\ u=0 & \text { on } \partial \Omega .\end{cases}
$$

The homogenized coefficients are given by

$$
\bar{D}_{i j}=\frac{1}{|Y|} \int_{Y} A(y) \psi^{2}(y)\left(\delta_{i j}-\frac{\partial \theta^{j}}{\partial y_{i}}(y)\right) d y \quad \text { and } \quad \bar{\sigma}=\frac{1}{|Y|} \int_{Y} \sigma(y) \psi^{2}(y) d y
$$


where the functions $\left(\theta^{j}\right)_{1 \leq j \leq N}$ are defined by

$$
\left\{\begin{array}{l}
-\operatorname{div}\left(A(y) \psi^{2}(y)\left(\nabla \theta^{j}+e_{j}\right)\right)=0 \quad \text { in } Y \\
y \rightarrow \theta^{j}(y) \quad Y-\text { periodic. }
\end{array}\right.
$$

In Theorem 3.1 the convergence of the eigenvectors hold up to the extraction of a subsequence because of a possible multiplicity of the limit eigenvalue. However, if the limit eigenvalue is simple (which is the case for the first one), then there is no need to extract a subsequence. The simplicity of the one-group case $K=1$ comes from the fact that it is a scalar self-adjoint problem.

In the multi-group case $K \geq 2$, system (8) is not self-adjoint. A simple generalization of Theorem 3.1 would be that the first direct and adjoint eigenvectors of the periodic cell problem control the oscillatory behavior of the eigenvector flux $\phi^{\epsilon}$. It turns out that this intuition is valid if the following symmetry condition is satisfied

$$
\sum_{\alpha=1}^{K} \int_{Y} A_{\alpha}(y)\left(\psi_{\alpha} \nabla \psi_{\alpha}^{*}-\psi_{\alpha}^{*} \nabla \psi_{\alpha}\right) d y=0,
$$

where $\left(\psi_{\alpha}\right)_{1 \leq \alpha \leq K}$ (resp. $\left.\left(\psi_{\alpha}^{*}\right)_{1 \leq \alpha \leq K}\right)$ are the components of the first eigenvector $\psi$ of (13) (resp. $\psi^{*}$ of (14)). Condition (18) is obviously fulfilled if system (8) were self-adjoint. As observed by Malige in [23], it is also verified if all cross sections and diffusion coefficients are symmetric functions in the unit cell $Y=[0,1]^{N}$ (more precisely, every coefficient should have a cubic symmetry, i.e. be symmetric with respect to all hyperplanes parallel to the axes and passing through the middle of the cell). Indeed, in such a case $\psi_{\alpha}$ and $\psi_{\alpha}^{*}$ have also cubic symmetry and each integral in (18) vanishes. However, it is not difficult to build, at least numerically, examples for which (18) does not hold (and Theorem 3.2 is clearly wrong).

Theorem 3.2 Assume that the symmetry condition (18) is satisfied.

Let $\left(\psi_{\alpha}\right)_{1 \leq \alpha \leq K}$ (resp. $\left.\left(\psi_{\alpha}^{*}\right)_{1 \leq \alpha \leq K}\right)$ be the components of the eigenvector $\psi$ of (13) (resp. $\psi^{*}$ of (14)) associated to the first eigenvalue $\mu^{\infty}$. Let $\left(\phi^{\epsilon, m}, \mu^{\epsilon, m}\right)$ be the $m^{\text {th }}$ eigenpair of system (8). Then,

$$
\begin{aligned}
& \phi_{\alpha}^{\epsilon, m}=u_{\alpha}^{\epsilon, m}(x) \psi_{\alpha}\left(\frac{x}{\epsilon}\right) \quad \forall \alpha \in\{1, \ldots, K\} \\
& \mu^{\epsilon, m}=\mu^{\infty}+\epsilon^{2} \nu^{m}+o\left(\epsilon^{2}\right)
\end{aligned}
$$

where, up to a subsequence, each component $u_{\alpha}^{\epsilon, m}$ converges weakly in $H_{0}^{1}(\Omega)$ to the same limit $u^{m}$ which is an eigenvector associated to the $m^{\text {th }}$ eigenvalue $\nu^{m}$ of the scalar homogenized problem

$$
\left\{\begin{aligned}
-\operatorname{div}(\bar{D} \nabla u(x)) & =\nu \bar{\sigma} u(x) & \text { in } \Omega \\
u & =0 & \text { on } \partial \Omega
\end{aligned}\right.
$$

The homogenized coefficients are

$$
\bar{\sigma}=\sum_{\alpha, \beta=1}^{K} \int_{Y} \sigma_{\alpha, \beta}(y) \psi_{\beta}(y) \psi_{\alpha}^{*}(y) d y,
$$


and $\bar{D}$ is a $N \times N$ positive definite matrix defined by its entries

$$
\begin{aligned}
\bar{D}_{i, j}= & \sum_{\alpha=1}^{K} \int_{Y} A_{\alpha} \psi_{\alpha} \psi_{\alpha}^{*} \nabla\left(y_{i}+\theta_{i, \alpha}\right) \nabla\left(y_{j}+\theta_{j, \alpha}\right) d y \\
& +\sum_{\alpha, \beta=1}^{K} \int_{Y} \frac{1}{2} \psi_{\alpha}^{*} \psi_{\beta}\left(\mu^{\infty} \sigma_{\alpha, \beta}-\Sigma_{\alpha, \beta}\right)\left(\theta_{i, \alpha}-\theta_{i, \beta}\right)\left(\theta_{j, \alpha}-\theta_{j, \beta}\right) d y
\end{aligned}
$$

where, for each $1 \leq i \leq N$, the components $\left(\theta_{i, \alpha}\right)_{1 \leq \alpha \leq K}$ are defined by

$$
\theta_{i, \alpha}=\frac{\zeta_{i, \alpha}}{\psi_{\alpha}}
$$

and $\zeta_{i}=\left(\zeta_{i, \alpha}\right)_{1 \leq \alpha \leq K}$ is the solution of

$$
\left\{\begin{array}{l}
-\operatorname{div}\left(A \nabla(y) \nabla \zeta_{i}\right)+\Sigma(y) \zeta_{i}=\mu^{\infty} \sigma(y) \zeta_{i}+Z_{i} \quad \text { in } Y \\
y \rightarrow \zeta_{i}(y) Y \text {-periodic }
\end{array}\right.
$$

where the right hand side $Z_{i}$ has components $Z_{i, \alpha}=\frac{1}{\psi_{\alpha}(y)} \operatorname{div}\left(A_{\alpha}(y) \psi_{\alpha}^{2}(y) \nabla y_{i}\right)$ for $1 \leq \alpha \leq K$.

Remark 3.3 The homogenized problem (19) has been formally found by Malige [23] using the heuristic method of two-scale asymptotic expansions. Theorem 3.2 justifies rigorously this result by furnishing a convergence proof.

It is interesting to notice that in the multigroup model, as long as a symmetry condition is fulfilled, the macroscopic behaviour is described by a single diffusion equation. This explain why one-energy-group models are still popular in reactor physics, where the symmetry condition is usually observed: the global trend of the power distribution in the reactor is indeed given by a homogenized one-energygroup model. The cost of such a simplification is merely a less accurate local description of the fluxes.

Remark 3.4 The convergence of the eigenvectors holds up to a subsequence because the corresponding homogenized eigenvalue may be multiple. However, for the first eigenvalue which is simple, a suitable normalization of the eigenvector shows that the entire sequence of eigenvectors converge.

Observe also that it was not proved that the original system (8) has an infinite number of eigenvalues. However, since the homogenized diffusion equation (19) does so, Theorem 3.2 proves that, as $\epsilon$ goes to 0 , the number of eigenvalues for (8), at least, converges to infinity.

The homogenized diffusion matrix $\bar{D}$, given in (21), may be defined by several different formulae (see Propositions 5.6 and 5.7) which are all equivalent, at least for the symmetric part of $\bar{D}$, which is the only relevant information in the diffusion equation (19).

Remark also that equation (22) is of the same type as the cell eigenvalue problem (13), but with a source term. Therefore, it admits a solution provided 
that the Fredholm alternative holds, i.e. the source term must be orthogonal to the adjoint first eigenvector $\psi^{*}$. This is precisely the symmetry condition (18).

Without the symmetry condition (18), we cannot hope to obtain a similar result as is shown by the next Proposition. However, a recent note [13] solves completely the case when (18) is not satisfied.

Proposition 3.5 Assume that the symmetry condition (18) is not fulflled. Let $\mu^{\epsilon, 1}$ be the first eigenvalue of system (8), and $\mu^{\infty}$ the first one of the cell problem (13). Then,

$$
\lim _{\epsilon \rightarrow 0} \frac{\mu^{\epsilon, 1}-\mu^{\infty}}{\epsilon^{2}}=+\infty
$$

We now turn to the proof of the above results. As already said, we begin with the one-group case which is much simpler.

Proof of Theorem 3.1. In the one-energy group case, equation (8) being self adjoint, its eigenvalues are characterized by the min-max formula

$$
\mu^{\epsilon, m}=\min _{\substack{W_{m} \subset H_{0}^{1}(\Omega) \\ \operatorname{dim} W_{m}=m}} \max _{\substack{\phi \in W_{m} \\ \phi \neq 0}} \frac{\epsilon^{2} \int_{\Omega} A\left(\frac{x}{\epsilon}\right)|\nabla \phi|^{2} d x+\int_{\Omega} \Sigma\left(\frac{x}{\epsilon}\right) \phi^{2} d x}{\int_{\Omega} \sigma\left(\frac{x}{\epsilon}\right) \phi^{2} d x} .
$$

For any function $\phi \in H_{0}^{1}(\Omega)$, we may define

$$
u(x)=\frac{\phi(x)}{\psi\left(\frac{x}{\epsilon}\right)},
$$

since the first eigenvector $\psi$ of the cell problem (13) is bounded from below by a positive constant (by virtue of Proposition 2.6 it is a continuous function on $Y$ and it is positive). A priori, $u$ defined by (23) belongs merely to $L^{2}(\Omega)$, but a simple computation shows that

$$
\frac{\epsilon^{2} \int_{\Omega} A\left(\frac{x}{\epsilon}\right)|\nabla \phi|^{2} d x+\int_{\Omega} \Sigma\left(\frac{x}{\epsilon}\right) \phi^{2} d x}{\int_{\Omega} \sigma\left(\frac{x}{\epsilon}\right) \phi^{2} d x}=\mu^{\infty}+\epsilon^{2} \frac{\int_{\Omega} A\left(\frac{x}{\epsilon}\right) \psi^{2}\left(\frac{x}{\epsilon}\right)|\nabla u|^{2} d x}{\int_{\Omega} \sigma\left(\frac{x}{\epsilon}\right) \psi^{2}\left(\frac{x}{\epsilon}\right) u^{2} d x}
$$

which proves that $u$ is indeed a function of $H_{0}^{1}(\Omega)$. Furthermore, this change of variables yields that if $\left(\mu^{\epsilon, m}, \phi^{\epsilon, m}\right)$ is the $m^{t h}$ eigenpair of $(8)$, then $\left(\nu^{\epsilon, m}, u^{\epsilon, m}\right)$, defined by

$$
\nu^{\epsilon, m}=\frac{\mu^{\epsilon, m}-\mu^{\infty}}{\epsilon^{2}} \text { and } u^{\epsilon, m}(x)=\frac{\phi^{\epsilon, m}(x)}{\psi\left(\frac{x}{\epsilon}\right)},
$$

is also the $m^{t h}$ eigenpair of

$$
\left\{\begin{array}{l}
-\operatorname{div}\left(D\left(\frac{x}{\epsilon}\right) \nabla u^{\epsilon}(x)\right)=\nu^{\epsilon} s\left(\frac{x}{\epsilon}\right) u^{\epsilon}(x) \text { in } \Omega \\
u^{\epsilon}=0 \text { on } \partial \Omega
\end{array}\right.
$$


where $D\left(\frac{x}{f}\right)=\psi^{2}\left(\frac{x}{f}\right) A\left(\frac{x}{f}\right)$, and $s\left(\frac{x}{f}\right)=\sigma\left(\frac{x}{f}\right) \psi^{2}\left(\frac{x}{f}\right)$. Because $\psi$ is bounded and strictly positive, $D$ and $s$ satisfy the same hypothesis as $A$ and $\sigma$. The homogenization of problem (24) is classical (see, for example [20]). Its eigenvalues $\nu^{\epsilon, m}$, labeled by increasing order, and the associated normalized eigenvectors $u^{\epsilon, m}$ satisfy

$$
\left(\nu^{\epsilon, m}, u^{\epsilon, m}\right) \rightarrow\left(\nu^{m}, u^{m}\right) \text { in } \mathbb{R} \times\left(H_{0}^{1}(\Omega) \text { weak }\right)
$$

where $\left(\nu^{m}, u^{m}\right)$ are the $m^{t h}$ eigenpair of the homogenized problem (15). The convergence of the eigenvectors hold up to a subsequence because of the possible multiplicity of the limit eigenvalue. $\square$.

We now focus on the proof of the homogenization process in the multi-group case $K \geq 1$. Our strategy is the following: we reduce the homogenization of the spectral problem to that of an equivalent system with a fixed source term. Then, upon admitting the homogenization results of Section 5 concerning the homogenization of this source problem, we prove all the above theorems. In order to simplify the notations, it is understood that we focus on a given (sub)sequence of eigenvalues with the same ordering $m$. Hence, indices $m$ will be dropped in the sequel.

Proposition 3.6 For $1 \leq \alpha \leq K$, let $T_{\alpha}$ and $T_{\alpha}^{*}$ be the following linear operators

$$
\begin{aligned}
& T_{\alpha}: H_{0}^{1}(\Omega) \rightarrow H_{0}^{1}(\Omega) \text { and } T_{\alpha}^{*}: H_{0}^{1}(\Omega) \rightarrow H_{0}^{1}(\Omega) \\
& \phi(x) \rightarrow \frac{\phi(x)}{\psi_{\alpha}\left(\frac{x}{\epsilon}\right)} \quad \phi(x) \rightarrow \frac{\phi(x)}{\psi_{\alpha}^{*}\left(\frac{x}{\epsilon}\right)}
\end{aligned}
$$

Then, $T_{\alpha}$ and $T_{\alpha}^{*}$ are bounded, bicontinuous operators.

Proof. By virtue of Corollary 2.5 and Proposition 2.6, we know that there exist positive constants $C>c>0$ such that $C>\psi_{\alpha}(y)>c$ for all $y \in Y$. Consequently, for all $\phi \in H_{0}^{1}(\Omega)$, defining $u=T_{\alpha}(\phi)$, we have

$$
C\|\phi\|_{L^{2}(\Omega)} \leq\|u\|_{L^{2}(\Omega)} \leq c\|\phi\|_{L^{2}(\Omega)} .
$$

Hence $T_{\alpha}$ is an homeorphism in $L^{2}(\Omega)$. On the other hand,

$$
\begin{aligned}
\int_{\Omega} A_{\alpha} \nabla \phi \cdot \nabla \phi & =\int_{\Omega} A_{\alpha} \nabla\left(u \psi_{\alpha}\right) \cdot \nabla\left(u \psi_{\alpha}\right) \\
& =\int_{\Omega} A_{\alpha}\left(\psi_{\alpha}\right)^{2} \nabla u \cdot \nabla u+\int_{\Omega} A_{\alpha} \nabla \psi_{\alpha} \cdot \nabla\left(u^{2} \psi_{\alpha}\right) .
\end{aligned}
$$

Using equation (13), defining $\psi$, yields

$\left|\int_{\Omega} A_{\alpha} \nabla \psi_{\alpha} \cdot \nabla\left(u^{2} \psi_{\alpha}\right)\right|=\frac{1}{\epsilon^{2}}\left|\sum_{\beta=1}^{K} \int_{\Omega}\left(-\Sigma_{\alpha, \beta}+\mu^{\infty} \sigma_{\alpha, \beta}\right) \psi_{\beta} \psi_{\alpha} u^{2}\right| \leq \frac{C}{\epsilon^{2}}\|u\|_{L^{2}(\Omega)}^{2}$ 
Hence, by coercivity and boundedness of $A_{\alpha}$, we deduce from (25) and (26) that there exists a constant $C>0$ such that

$$
\begin{gathered}
\frac{1}{C}\left(\|\phi\|_{H_{0}^{1}(\Omega)}^{2}-\frac{1}{\epsilon^{2}}\left\|T_{\alpha}(\phi)\right\|_{L^{2}(\Omega)}^{2}\right) \leq\left\|T_{\alpha}(\phi)\right\|_{H_{0}^{1}(\Omega)}^{2} \\
\left\|T_{\alpha}(\phi)\right\|_{H_{0}^{1}(\Omega)}^{2} \leq C\left(\|\phi\|_{H_{0}^{1}(\Omega)}^{2}+\frac{1}{\epsilon^{2}}\left\|T_{\alpha}(\phi)\right\|_{L^{2}(\Omega)}^{2}\right),
\end{gathered}
$$

which concludes the proof for $T_{\alpha}$. The proof for $T_{\alpha}^{*}$ is similar.

Proposition 3.7 The multigroup eigenvalue problem (8) is equivalent to the following eigenvalue problem

$$
\left\{\begin{array}{l}
-\operatorname{div}\left(D\left(\frac{x}{\epsilon}\right) \nabla u^{\epsilon}\right)+\frac{1}{\epsilon^{2}} Q^{\epsilon}\left(u^{\epsilon}\right)=\nu^{\epsilon} B\left(\frac{x}{\epsilon}\right) u^{\epsilon} \text { in } \Omega, \\
u^{\epsilon} \in H_{0}^{1}(\Omega)^{K}
\end{array}\right.
$$

where the components $\left(u_{\alpha}^{\epsilon}\right)_{1 \leq \alpha \leq K}$ of $u^{\epsilon}$ are defined by

$$
u_{\alpha}^{\epsilon}(x)=\frac{\phi_{\alpha}^{\epsilon}(x)}{\psi_{\alpha}\left(\frac{x}{\epsilon}\right)},
$$

the eigenvalue $\nu^{\epsilon}$ is defined by

$$
\nu^{\epsilon}=\frac{\mu^{\epsilon}-\mu^{\infty}}{\epsilon^{2}},
$$

$D(y)$ is a $Y$-periodic fourth-order tensor which is block diagonal, i.e. $D=$ $\operatorname{diag}\left(D_{1}, \ldots, D_{K}\right)$ with

$$
D_{\alpha}(y)=\psi_{\alpha}(y) \psi_{\alpha}^{*}(y) A_{\alpha}(y) \quad \forall \alpha \in 1, \ldots, K,
$$

$B$ is a $K \times K Y$-periodic matrix with entries

$$
B_{\alpha, \beta}(y)=\sigma_{\alpha, \beta}(y) \psi_{\beta}(y) \psi_{\alpha}^{*}(y)
$$

and $Q^{\epsilon}$ is a continuous linear operator from $H_{0}^{1}(\Omega)^{K}$ into $H^{-1}(\Omega)^{K}$, defined by (36). Furthermore, there exist two positive constants $C>c>0$ (independent of $\epsilon$ ) such that, for any $u \in H_{0}^{1}(\Omega)^{K}$,

$$
C \sum_{\alpha, \beta=1}^{K}\left\|u_{\alpha}-u_{\beta}\right\|_{L^{2}(\Omega)}^{2} \geq \int_{\Omega} Q^{\epsilon}(u) \cdot u d x \geq c \sum_{\alpha, \beta=1}^{K}\left\|u_{\alpha}-u_{\beta}\right\|_{L^{2}(\Omega)}^{2} .
$$

Remark 3.8 If we take into account Remark 2.1, and allow cross-sections to be positive merely on $Y_{0} \subset \subset Y$, then Proposition 3.7 is true if (30) is replaced by

$$
C \sum_{\alpha, \beta=1}^{K}\left\|u_{\alpha}-u_{\beta}\right\|_{L^{2}\left(\Omega_{\epsilon}\right)}^{2} \geq \int_{\Omega} Q^{\epsilon}(u) \cdot u d x \geq c \sum_{\alpha, \beta=1}^{K}\left\|u_{\alpha}-u_{\beta}\right\|_{L^{2}\left(\Omega_{\epsilon}\right)}^{2}
$$


where $\Omega_{\epsilon}$ is a periodic domain defined by

$$
\Omega_{\epsilon}=\bigcup_{i=1}^{N(\epsilon)} Y_{0, \epsilon}^{i} \cap \Omega
$$

with $\left(Y_{0, \epsilon}^{i}\right)_{i=1, \ldots, N(\epsilon)}$ the collection of homothetics of $Y_{0}$, corresponding to a cubic mesh of size $\epsilon$ covering $\Omega$, where cross-sections are positive.

Proof. Let us first prove that $u^{\epsilon}$ defined by (28) is a solution of equation (27). We write the variational formulation of (8), factorizing its solution $\phi^{\epsilon}$ in $u_{\alpha}^{\epsilon}(x) \psi_{\alpha}\left(\frac{x}{\epsilon}\right)$ and the test function in $v_{\alpha}(x) \psi_{\alpha}^{*}\left(\frac{x}{\epsilon}\right)$,

$$
\begin{gathered}
\epsilon^{2} \sum_{\alpha=1}^{K} \int_{\Omega} A_{\alpha}\left(\frac{x}{\epsilon}\right) \nabla\left(u_{\alpha}^{\epsilon} \psi_{\alpha}\right) \cdot \nabla\left(v_{\alpha} \psi_{\alpha}^{*}\right)+\sum_{\alpha, \beta=1}^{K} \int_{\Omega} \Sigma_{\alpha, \beta}\left(\frac{x}{\epsilon}\right) u_{\alpha}^{\epsilon} \psi_{\alpha} v_{\alpha} \psi_{\alpha}^{*}= \\
\mu^{\epsilon} \sum_{\alpha, \beta=1}^{K} \int_{\Omega} \sigma_{\alpha, \beta}\left(\frac{x}{\epsilon}\right) u_{\alpha}^{\epsilon} \psi_{\alpha} v_{\alpha} \psi_{\alpha}^{*} .
\end{gathered}
$$

Remark that this factorization is licit by virtue of Proposition 3.6. Developing the above equation yields

$$
\int_{\Omega} D\left(\frac{x}{\epsilon}\right) \nabla u^{\epsilon} \cdot \nabla v+\frac{1}{\epsilon^{2}} q\left(u^{\epsilon}, \nabla u^{\epsilon}, v, \nabla v\right)=\nu^{\epsilon} \int_{\Omega} B\left(\frac{x}{\epsilon}\right) u^{\epsilon} \cdot v,
$$

where $v$ is a function in $H_{0}^{1}(\Omega)^{K}$ of components $\left(v_{\alpha}\right)_{1 \leq \alpha \leq K}$, and $q$ is defined by

$$
\begin{gathered}
q\left(u^{\epsilon}, \nabla u^{\epsilon}, v, \nabla v\right)=\epsilon^{2} \sum_{\alpha=1}^{K} \int_{\Omega} A_{\alpha} \psi_{\alpha} v_{\alpha} \nabla \psi_{\alpha}^{*} \cdot \nabla u_{\alpha}^{\epsilon}+\sum_{\alpha, \beta=1}^{K} \int_{\Omega} \Sigma_{\alpha, \beta} \psi_{\alpha} u_{\alpha}^{\epsilon} v_{\alpha} \psi_{\alpha}^{*} \\
+\epsilon^{2} \sum_{\alpha=1}^{K} \int_{\Omega} A_{\alpha} u_{\alpha}^{\epsilon} v_{\alpha} \nabla \psi_{\alpha} \cdot \nabla \nabla \psi_{\alpha}^{*}+\epsilon^{2} \sum_{\alpha=1}^{K} \int_{\Omega} A_{\alpha} u_{\alpha}^{\epsilon} \psi_{\alpha}^{*} \nabla \psi_{\alpha} \cdot \nabla v_{\alpha} \\
-\sum_{\alpha, \beta=1}^{K} \mu^{\infty} \int_{\Omega} \sigma_{\alpha, \beta} \psi_{\beta} \psi_{\alpha}^{*} u_{\beta}^{\epsilon} v_{\alpha} .
\end{gathered}
$$

The last four terms in (33) also arise in the variational formulation of the periodic eigenvalue problem (13), defining $\psi$, rescaled to size $\epsilon$ with the test function $\left(v_{\alpha} u_{\alpha}^{\epsilon} \psi_{\alpha}^{*}\right)_{\alpha}$. Using this variational formulation we obtain, after some algebra, a simplified formula for $q$

$$
q\left(u^{\epsilon}, \nabla u^{\epsilon}, v, \nabla v\right)=\epsilon \sum_{\alpha=1}^{K} \int_{\Omega} v_{\alpha} J_{\alpha}\left(\frac{x}{\epsilon}\right) \cdot \nabla u_{\alpha}^{\epsilon}+\int_{\Omega} \tilde{Q}\left(\frac{x}{\epsilon}\right) u^{\epsilon} \cdot v
$$

with

$$
J_{\alpha}(y)=A_{\alpha}(y)\left(\psi_{\alpha}(y) \nabla_{y} \psi_{\alpha}^{*}(y)-\psi_{\alpha}^{*}(y) \nabla_{y} \psi_{\alpha}(y)\right)
$$


and $\tilde{Q}$ is the $Y$-periodic $K \times K$ matrix defined by its entries

$$
\begin{aligned}
& \tilde{Q}_{\alpha, \beta}(y)=\left(\Sigma_{\alpha, \beta}(y)-\mu^{\infty} \sigma_{\alpha, \beta}(y)\right) \psi_{\beta}(y) \psi_{\alpha}^{*}(y) \leq 0 \text { if } \alpha \neq \beta, \\
& \tilde{Q}_{\alpha, \alpha}(y)=-\sum_{\substack{\beta=1 \\
\beta \neq \alpha}}^{K} \tilde{Q}_{\alpha, \beta}(y) \geq 0 .
\end{aligned}
$$

Therefore, $q$ can be rewritten

$$
q\left(u^{\epsilon}, \nabla u^{\epsilon}, v, \nabla v\right)=\int_{\Omega} Q^{\epsilon}(u) \cdot v d x,
$$

where, upon defining a second order tensor $J$ with lines $J_{\alpha}$, the operator $Q^{\epsilon}$ is defined by

$$
Q^{\epsilon}(u)=\epsilon J\left(\frac{x}{\epsilon}\right) \cdot \nabla u+\tilde{Q}\left(\frac{x}{\epsilon}\right) u .
$$

The matrix $\tilde{Q}$ is clearly bounded, but it is not clear whether $J$ belongs or not to $L^{\infty}(\Omega)$. Thus, in order to prove that $Q^{\epsilon}$ is continuous, we have to rely on Proposition 3.6. Introducing homeomorphisms $P$ and $P^{*}$ defined by,

$$
\begin{aligned}
& P:\left(H_{0}^{1}(\Omega)\right)^{K} \rightarrow\left(H_{0}^{1}(\Omega)\right)^{K} \text { and } P^{*}:\left(H_{0}^{1}(\Omega)\right)^{K} \rightarrow\left(H_{0}^{1}(\Omega)\right)^{K} \\
& u_{\alpha} \quad \rightarrow T_{\alpha}^{-1} u_{\alpha} \quad u_{\alpha} \quad \rightarrow\left(T_{\alpha}^{*}\right)^{-1} u_{\alpha}
\end{aligned}
$$

the above computation in reverse order shows that

$$
\begin{aligned}
\int_{\Omega} Q^{\epsilon}(u) \cdot v d x & =\epsilon^{2} \int_{\Omega} A\left(\frac{x}{\epsilon}\right) \nabla(P u) \cdot \nabla\left(P^{*} v\right)-\epsilon^{2} \int_{\Omega} D\left(\frac{x}{\epsilon}\right) \nabla u \cdot \nabla v \\
& +\int_{\Omega} \Sigma\left(\frac{x}{\epsilon}\right) P u \cdot P^{*} v-\mu^{\infty} \int_{\Omega} B\left(\frac{x}{\epsilon}\right) u \cdot v \\
& \leq C\|u\|_{H^{1}(\Omega)^{K}}\|v\|_{H^{1}(\Omega)^{K}}
\end{aligned}
$$

which proves that $Q^{\epsilon}$ is bounded and continuous from $H_{0}^{1}(\Omega)^{K}$ into $H^{-1}(\Omega)^{K}$. Finally, to obtain inequalities (30), we remark that the cell eigenvalue problems (13) and (14) implies, for any $1 \leq \alpha \leq K$,

$$
-\operatorname{div}_{y} J_{\alpha}(y)+\sum_{\beta=1}^{K} \tilde{Q}_{\beta, \alpha}(y)=\sum_{\beta=1}^{K} \tilde{Q}_{\alpha, \beta}(y) .
$$

Multiplying (37) by $u_{\alpha}^{2}$, we deduce

$$
\sum_{\alpha=1}^{K} \int_{\Omega} u_{\alpha} J_{\alpha} \cdot \nabla u_{\alpha}=\frac{1}{2} \sum_{\alpha, \beta=1}^{K} \int_{\Omega}\left(\tilde{Q}_{\alpha, \beta} u_{\alpha}^{2}-\tilde{Q}_{\beta, \alpha} u_{\alpha}^{2}\right)
$$


Therefore, using (36) and (35), we obtain

$$
\begin{aligned}
\int_{\Omega} Q^{\epsilon}(u) \cdot u d x & =\sum_{\alpha, \beta=1}^{K} \int_{\Omega}\left(\tilde{Q}_{\alpha, \beta}\left(\frac{x}{\epsilon}\right)\left(u_{\beta} u_{\alpha}-u_{\alpha} u_{\alpha}+\frac{1}{2} u_{\alpha}^{2}\right)-\frac{1}{2} \tilde{Q}_{\beta, \alpha}\left(\frac{x}{\epsilon}\right) u_{\alpha}^{2}\right) \\
& =-\frac{1}{2} \sum_{\alpha, \beta=1}^{K} \int_{\Omega} \tilde{Q}_{\alpha, \beta}\left(\frac{x}{\epsilon}\right)\left(u_{\alpha}-u_{\beta}\right)^{2}
\end{aligned}
$$

Remark that, in view of (10), for all $\alpha \neq \beta, \tilde{Q}_{\alpha, \beta} \leq 0$ and, since $|\alpha-\beta|=1$ implies $-\tilde{Q}_{\alpha, \beta}>q>0$,

$$
\int_{\Omega} Q^{\epsilon}(u) \cdot u d x \geq \frac{q}{2} \sum_{\alpha=1}^{K-1}\left\|u_{\alpha}-u_{\alpha+1}\right\|^{2}+\frac{q}{2}\left\|u_{1}-u_{K}\right\|^{2} \geq \frac{q}{2 K^{2}} \sum_{\alpha, \beta=1}^{K}\left\|u_{\alpha}-u_{\beta}\right\|^{2} \geq 0
$$

which is the desired result. $\square$.

Unlike in the one-group case (see Theorem 3.1), the multi-group problem (27) is not self-adjoint. Therefore, we can not use the min-max principle to characterize the eigenvalues. Rather, we associate to this equation a linear operator $S_{\epsilon}$. Studying the convergence of $S_{\epsilon}$ will allow us to deduce a convergence result for the spectrum of $(27)$. Let us define a linear operator $S_{\epsilon}$ by

$$
\begin{array}{ll}
S_{\epsilon}: L^{2}(\Omega)^{K} & \rightarrow L^{2}(\Omega)^{K} \\
f=\left(f_{\alpha}\right)_{1 \leq \alpha \leq K} & \rightarrow u=\left(u_{\alpha}\right)_{1 \leq \alpha \leq K} \text { unique solution of } \\
& \left\{\begin{array}{l}
-\operatorname{div}\left(D\left(\frac{x}{\epsilon}\right) \nabla u\right)+\frac{1}{\epsilon^{2}} Q^{\epsilon}(u)=f \text { in } \Omega, \\
u=0 \text { on } \partial \Omega .
\end{array}\right.
\end{array}
$$

Remark that the eigenvalue problem (27) can be rewritten

$$
\left(S_{\epsilon}\right)^{-1} u^{\epsilon}=\nu^{\epsilon} B\left(\frac{x}{\epsilon}\right) u^{\epsilon}
$$

Lemma 3.9 For any fixed $\epsilon>0, S_{\epsilon}$ is a linear compact operator in $L^{2}(\Omega)^{K}$.

Proof. We proved in Proposition 3.7 that $Q^{\epsilon}$ is a continuous operator from $H_{0}^{1}(\Omega)^{K}$ into $H^{-1}(\Omega)^{K}$, such that $\int_{\Omega} Q^{\epsilon}(u) \cdot u d x \geq 0$. On the other hand, the diffusion tensor $D$ satisfies the same type of assumptions than $A$. Hence the left hand side of (39) defines a continuous and coercive bi-linear form in its variational formulation. Then, the Lax-Milgram lemma shows that (39) has a unique solution, i.e. $S_{\epsilon}$ is well defined. The compact embedding of $H_{0}^{1}(\Omega)$ in $L^{2}(\Omega)$ gives the compactness of $S_{\epsilon}$.

In section 5 we shall prove the following

Proposition 3.10 Let $f^{\epsilon}$ be a sequence which converges weakly in $L^{2}(\Omega)^{K}$ to $f=\left(f_{\alpha}\right)_{1 \leq \alpha \leq K}$. Then, the sequence $u^{\epsilon}=S_{\epsilon}\left(f^{\epsilon}\right)$ converges weakly in $H_{0}^{1}(\Omega)^{K}$ 
to $\left(u^{0}, \ldots, u^{0}\right)$ which is defined by $u^{0}=S\left(\sum_{\alpha=1}^{K} f_{\alpha}\right)$.

If the symmetry condition (18) is not satisfied, then $S=0$. If the symmetry condition (18) is satisfied, $S$ is the following compact operator

$$
\begin{aligned}
& S: L^{2}(\Omega) \rightarrow L^{2}(\Omega) \\
& f \rightarrow u \text { unique solution of } \\
& \left\{\begin{array}{l}
-\operatorname{div}(\bar{D} \nabla u(x))=f \text { in } \Omega, \\
u=0 \text { on } \partial \Omega,
\end{array}\right.
\end{aligned}
$$

where $\bar{D}$ is the constant positive definite matrix defined by (21) (see also Proposition 5.6).

Upon admitting, for the moment, Proposition 3.10, we are in a position to prove our main results.

Proof of Theorem 3.2 and Proposition 3.5. Remark that Proposition 3.10 implies that the sequence of operators $S_{\epsilon}$, defined by (39), uniformly converges to the limit operator defined in $L^{2}(\Omega)^{K}$ by

$$
f=\left(f_{\alpha}\right)_{1 \leq \alpha \leq K} \rightarrow\left(S\left(\sum_{\alpha=1}^{K} f_{\alpha}\right), \ldots, S\left(\sum_{\alpha=1}^{K} f_{\alpha}\right)\right) .
$$

The asymptotic analysis of the eigenvalue problem (27) is truely controled by the convergence of the sequence of operators $T_{\epsilon}$ defined by

$$
\begin{array}{ll}
T_{\epsilon}: L^{2}(\Omega)^{K} & \rightarrow L^{2}(\Omega)^{K} \\
f=\left(f_{\alpha}\right)_{1 \leq \alpha \leq K} & \rightarrow S_{\epsilon}\left(B\left(\frac{x}{\epsilon}\right) f\right)
\end{array}
$$

Namely, the eigenvalues of $T_{\epsilon}$ are inverse of those of (27). Introducing the averages $\bar{B}_{\alpha, \beta}=\int_{Y} B_{\alpha, \beta}(y) d y$ which are the weak limits of the entries of the matrix $B\left(\frac{x}{\epsilon}\right)$, we define a limit operator $T$ by

$$
\begin{array}{ll}
T: L^{2}(\Omega)^{K} & \rightarrow L^{2}(\Omega)^{K} \\
f=\left(f_{\alpha}\right)_{1 \leq \alpha \leq K} & \rightarrow\left(S\left(\sum_{\alpha, \beta=1}^{K} \bar{B}_{\alpha, \beta} f_{\beta}\right), \ldots, S\left(\sum_{\alpha, \beta=1}^{K} \bar{B}_{\alpha, \beta} f_{\beta}\right)\right)
\end{array}
$$

The sequence $T_{\epsilon}$ converges ponctually to $T$, but usually not uniformly. However, Proposition 3.10 implies that the sequence of operators $T_{\epsilon}$ is collectively compact (see e.g. [6], [14]) in the sense that

$$
\left\{\begin{array}{l}
\forall f \in L^{2}(\Omega)^{K} \quad \lim _{\epsilon \rightarrow 0}\left\|T_{\epsilon}(f)-T(f)\right\|_{L^{2}(\Omega)^{K}}=0 \\
\text { The set }\left\{T_{\epsilon}(f):\|f\|_{L^{2}(\Omega)^{K}} \leq 1, \epsilon \geq 0\right\} \quad \text { is sequentially compact }
\end{array}\right.
$$

Then, as a consequence of Theorem 3.11 below, the $m^{\text {th }}$ eigenvalue of $T_{\epsilon}$ converges to the $m^{t h}$ eigenvalue of $T$ (counted with their multiplicity). This is precisely the content of Theorem 3.2. In the particular case when $S=0, T_{\epsilon}$ converges to 0 , and so does all its eigenvalues, which yields Theorem 3.5. 
Theorem 3.11 (see e.g. [6], [14]) Let $T_{n}$ be a sequence of compact operators that converges to $T$. Assume that $\left(T_{n}\right)_{n>1}$ is collectively compact and $T$ is compact. Let $\mu \in \mathbb{C}$ be an eigenvalue of $T$, of multiplicity $m$. Let $\Gamma$ be a smooth curve enclosing $\mu$ in the complex plane and leaving outside the rest of the spectrum of $T$. Then, for sufficiently large values of $n, \Gamma$ encloses also exactly $m$ eigenvalues of $T_{n}$ and leaves outside the rest of the spectrum of $T_{n}$.

\section{A priori estimates}

This section is devoted to establishing a priori estimates and recalling twoscale convergence results (see [1], [26]). In the sequel $f^{\epsilon}=\left(f_{\alpha}^{\epsilon}\right)_{1 \leq \alpha \leq K}$ denotes a bounded sequence in $L^{2}(\Omega)^{K}$, and $u^{\epsilon}=S_{\epsilon}\left(f^{\epsilon}\right)$ is the unique solution in $H_{0}^{1}(\Omega)^{K}$ of

$$
\left\{\begin{array}{l}
-\operatorname{div}\left(D\left(\frac{x}{\epsilon}\right) \nabla u^{\epsilon}\right)+\frac{1}{\epsilon^{2}} Q^{\epsilon}\left(u^{\epsilon}\right)=f^{\epsilon} \text { in } \Omega, \\
u^{\epsilon}=0 \text { on } \partial \Omega,
\end{array}\right.
$$

where $Q^{\epsilon}$ is a bounded linear operator from $H_{0}^{1}(\Omega)^{K}$ into $H^{-1}(\Omega)^{K}$, defined by (36), satisfying estimate (30).

Lemma 4.1 The solution $u^{\epsilon}$ of (40) satisfies the following estimate

$$
\sum_{\alpha=1}^{K}\left\|u_{\alpha}^{\epsilon}\right\|_{H_{0}^{1}(\Omega)}+\frac{1}{\epsilon} \sum_{\alpha, \beta=1}^{K}\left\|u_{\alpha}^{\epsilon}-u_{\beta}^{\epsilon}\right\|_{L^{2}(\Omega)} \leq C \sum_{\alpha=1}^{K}\left\|f_{\alpha}^{\epsilon}\right\|_{L^{2}(\Omega)}
$$

where $C>0$ is a positive constant independent of $\epsilon$.

Proof. Multiplying equation (40) by $u^{\epsilon}$, integrating by parts and using Poincaré inequality yields

$$
\sum_{\alpha=1}^{K}\left(\int_{\Omega} D_{\alpha}\left(\frac{x}{\epsilon}\right) \nabla u_{\alpha}^{\epsilon} \cdot \nabla u_{\alpha}^{\epsilon}\right)^{\frac{1}{2}}+\frac{1}{\epsilon}\left(\int_{\Omega} Q^{\epsilon}\left(u^{\epsilon}\right) \cdot u^{\epsilon}\right)^{\frac{1}{2}} \leq C \sum_{\alpha=1}^{K}\left\|f_{\alpha}^{\epsilon}\right\|_{L^{2}(\Omega)}
$$

Estimate (30) satisfied by $Q^{\epsilon}$ show that there exists a positive constant $c>0$ such that

$$
c \sum_{\alpha, \beta=1}^{K}\left\|u_{\alpha}-u_{\beta}\right\|_{L^{2}(\Omega)}^{2} \leq \int_{\Omega} Q^{\epsilon}(u) \cdot u d x,
$$

and the coercivity of the matrices $\left(D_{\alpha}\right)_{1 \leq \alpha \leq K}$ allow us to conclude.

Remark 4.2 The a priori estimates (41) are still valid when the cross-sections are not assumed positive everywhere in $Y$, but only on a sub-domain $Y_{0}$. Introducing the periodic domain $\Omega_{\epsilon}$, defined by (32), we have

$$
\sum_{\alpha=1}^{K}\left\|u_{\alpha}^{\epsilon}\right\|_{H_{0}^{1}(\Omega)}+\frac{1}{\epsilon} \sum_{\alpha, \beta=1}^{K}\left\|u_{\alpha}^{\epsilon}-u_{\beta}^{\epsilon}\right\|_{L^{2}\left(\Omega_{\epsilon}\right)} \leq C \sum_{\alpha=1}^{K}\left\|f_{\alpha}^{\epsilon}\right\|_{L^{2}(\Omega)} .
$$


A classical inequality in the theory of porous media homogenization (see e.g. [19]) states that

$$
\left\|u_{\alpha}^{\epsilon}-u_{\beta}^{\epsilon}\right\|_{L^{2}(\Omega)} \leq C\left(\left\|u_{\alpha}^{\epsilon}-u_{\beta}^{\epsilon}\right\|_{L^{2}\left(\Omega_{\epsilon}\right)}+\epsilon\left\|\nabla\left(u_{\alpha}^{\epsilon}-u_{\beta}^{\epsilon}\right)\right\|_{L^{2}(\Omega)^{N}}\right) .
$$

where $C$ is a positive constant independent of $\epsilon$. Then, a combination of (42) and (43) is equivalent to (41).

Remark 4.3 If we allow a small perturbation of size $\epsilon^{2}$ to the absorption section, that is, if $\Sigma\left(\frac{x}{\epsilon}\right)$ is replaced by $\Sigma\left(\frac{x}{\epsilon}\right)+\epsilon^{2} \Sigma^{\prime}\left(x, \frac{x}{\epsilon}\right)$, these a priori estimates are valid if and only if, for any $u \in L^{2}(\Omega)^{K}$,

$$
\sum_{\alpha, \beta=1}^{K} \int_{\Omega} \Sigma_{\alpha, \beta}^{\prime}\left(x, \frac{x}{\epsilon}\right) \psi_{\beta}\left(\frac{x}{\epsilon}\right) \psi_{\alpha}^{*}\left(\frac{x}{\epsilon}\right) u_{\alpha}(x) u_{\beta}(x) d x \geq 0 .
$$

Obviously, this condition is fulfilled if $\Sigma^{\prime}$ a positive diagonal matrix. In the general case, one needs to compute the first eigenvectors $\psi$ and $\psi^{*}$ of the cell eigenvalue problems (13) and (14) to know which perturbations are admissible.

Let us introduce some notations that we shall use in the definition of the two-scale convergence. We denote by $C_{\#}(Y)$ the space of continuous functions in $\mathbb{R}^{N}$ that are periodic of period $Y$, and $L_{\#}^{2}(Y)$ (respectively, $H_{\#}^{1}(Y)$ ) the subspace of $L^{2}\left(\mathbb{R}^{N}\right)$ (respectively, $H^{1}\left(\mathbb{R}^{N}\right)$ ) made of $Y$-periodic functions. We recall the main result of two-scale convergence (see [1], [26]).

\section{Proposition 4.4}

1. Let $u_{\epsilon}$ be a bounded sequence in $L^{2}(\Omega)$. There exist a subsequence, still denoted by $\epsilon$, and a limit $u_{0}(x, y) \in L^{2}\left(\Omega ; L_{\#}^{2}(Y)\right)$ such that $u_{\epsilon}$ two-scale converges to $u_{0}$ in the sense that

$$
\lim _{\epsilon \rightarrow 0} \int_{\Omega} u_{\epsilon}(x) \phi\left(x, \frac{x}{\epsilon}\right) d x=\int_{\Omega} \int_{Y} u_{0}(x, y) \phi(x, y) d x d y
$$

for all functions $\phi(x, y) \in L^{2}\left(\Omega ; C_{\#}(Y)\right)$.

2. Let $u_{\epsilon}$ be a bounded sequence in $H_{0}^{1}(\Omega)$. There exist a subsequence, still denoted by $\epsilon$, and limits $u(x) \in H_{0}^{1}(\Omega), u_{1}(x, y) \in L^{2}\left(\Omega ; H_{\#}^{1}(Y) / \mathbb{R}\right)$ such that $u_{\epsilon}$ converges weakly to $u(x)$ in $H_{0}^{1}(\Omega)$, and $\nabla u_{\epsilon}$ two-scale converges to $\nabla_{x} u(x)+\nabla_{y} u_{1}(x, y)$.

We also need a new lemma on two-scale convergence.

Lemma 4.5 Let $u_{\epsilon}$ be a bounded sequence in $H_{0}^{1}(\Omega)$, which converges weakly to $u(x)$ in $H_{0}^{1}(\Omega)$, and such that $\epsilon^{-1}\left(u_{\epsilon}-u\right)$ is uniformly bounded in $L^{2}(\Omega)$. Then there exists $u_{1}(x, y) \in L^{2}\left(\Omega ; H_{\#}^{1}(Y)\right)$ such that, up to a subsequence,

$$
\begin{array}{ll}
\nabla\left(u_{\epsilon}-u\right) & \rightarrow \nabla_{y} u_{1}(x, y) \\
\frac{1}{\epsilon}\left(u_{\epsilon}-u\right) & \rightarrow u_{1}(x, y)
\end{array}
$$

in the sense of two-scale convergence. 
Proof. Since $\epsilon^{-1}\left(u_{\epsilon}-u\right)$ is bounded in $L^{2}(\Omega)$, up to a subsequence, it twoscale converges to a limit $u^{*}(x, y)$. On the other hand, up to a subsequence, $\nabla u_{\epsilon}$ two-scale converges to $\nabla_{x} u(x)+\nabla_{y} u_{1}(x, y)$ with $u_{1}(x, y) \in L^{2}\left(\Omega ; H_{\#}^{1}(Y)\right)$. Therefore, for any test function $\psi(x, y) \in \mathcal{D}(\Omega \times Y)^{N}$, an integration by parts leads to

$$
\begin{aligned}
\lim _{\epsilon \rightarrow 0} \int_{\Omega}\left(\nabla u_{\epsilon}-\nabla u\right) \psi\left(x, \frac{x}{\epsilon}\right) d x & =\int_{\Omega} \int_{Y} \nabla_{y} u_{1}(x, y) \psi(x, y) d x d y \\
& =-\int_{\Omega} \int_{Y} u^{*}(x, y) \operatorname{div}_{y} \psi(x, y) d x d y
\end{aligned}
$$

We deduce that $\nabla_{y}\left(u_{1}-u^{*}\right)=0$, which implies that $u_{1}$ and $u^{*}$ differs by a function of $x$ only. Since the limit $u_{1}$ is defined up to a function of $x$ (only its gradient with respect to $y$ plays a role in Proposition 4.4), we can choose it to be equal to $u^{*}$.

In what follows, we shall use the notation $\mathbb{I}=\{1, \ldots, 1\} \in \mathbb{R}^{K}$. Then, if $u$ is a scalar function, $u \mathbb{I}$ denotes the vector-valued function with $K$ components equal to $u$, and $\mathbb{I} \otimes \nabla u$ denotes the $K \times N$ matrix with entries $\left(\partial u / \partial x_{i}\right)_{1 \leq \alpha<K, 1<i<N}$. Similarly, if $v$ is a vector in $\mathbb{R}^{K}$, we denote by $\mathbb{I}(v) \in \mathbb{R}^{K}$ its projection on the vector $\mathbb{I}$, i.e.,

$$
\mathbb{I}(v)=\left(\frac{1}{K} \sum_{\alpha=1}^{K} v_{\alpha}\right) \mathbb{I} .
$$

Finally, we define a Hilbert space $\mathcal{H}(Y)$ by

$$
\mathcal{H}(Y)=H_{\#}^{1}(Y)^{K} /(\mathbb{R} \times \mathbb{I})
$$

which is the quotient space of $H_{\#}^{1}(Y)^{K}$ by the subspace of constant vectors parallel to $\mathbb{I}$.

Proposition 4.6 Let $u^{\epsilon}$ be a sequence satisfying the a priori estimates (41) of Lemma 4.1. There exist a subsequence and limits $u^{0}(x) \in H_{0}^{1}(\Omega), u^{1}(x, y) \in$ $L^{2}(\Omega ; \mathcal{H}(Y))$ such that, for this subsequence, $u^{\epsilon}(x)$ converges weakly to $u^{0}(x) \mathbb{I}$ in $H_{0}^{1}(\Omega)^{K}$ and

$$
\begin{array}{ll}
\nabla u^{\epsilon} & \rightarrow \mathbb{I} \otimes \nabla_{x} u^{0}(x)+\nabla_{y} u^{1}(x, y) \\
\frac{1}{\epsilon}\left(u^{\epsilon}-\mathbb{I}\left(u^{\epsilon}\right)\right) & \rightarrow u^{1}(x, y)-\mathbb{I}\left(u^{1}\right)(x, y)
\end{array}
$$

in the sense of two-scale convergence.

Proof. Estimate (41) in Lemma 4.1 shows that $u^{\epsilon}$ is bounded in $H_{0}^{1}(\Omega)^{K}$. Therefore, there exists a limit $\left(u_{\alpha}^{0}\right)_{1 \leq \alpha \leq K}$ such that, up to a subsequence, for all $\alpha \in\{1, . ., K\}, u_{\alpha}^{\epsilon}$ converges weakly to $u_{\alpha}^{0}$ in $H_{0}^{1}(\Omega)$. From Proposition 4.4 we also know that there exists $\tilde{u}_{\alpha}^{1}(x, y) \in L^{2}\left(\Omega ; H_{\#}^{1}(Y) / \mathbb{R}\right)$ such that, up to a subsequence, $\nabla u_{\alpha}^{\epsilon}$ two-scale converges to $\nabla_{x} u_{\alpha}^{0}(x)+\nabla_{y} \tilde{u}_{\alpha}^{1}(x, y)$. Since (41) 
implies that $\epsilon^{-1}\left\|u_{\alpha}^{\epsilon}-u_{\beta}^{\epsilon}\right\|_{L^{2}(\Omega)}$ is also bounded for any $\alpha, \beta \in\{1, . ., K\}$, we deduce that all limit components coincide, i.e. $u_{\alpha}^{0}=u^{0}$ for any $\alpha \in\{1, . ., K\}$, namely $u^{\epsilon}$ converges weakly to $u^{0}(x) \mathbb{I}$ in $H_{0}^{1}(\Omega)^{K}$.

Furthermore, (41) implies that $\epsilon^{-1}\left\|u_{\alpha}^{\epsilon}-K^{-1} \sum_{\beta=1}^{K} u_{\beta}^{\epsilon}\right\|_{L^{2}(\Omega)}$ is also bounded. Then, arguing as in Lemma 4.5 , one can show that, for each $\alpha \in\{1, \ldots, K\}$, there exists a function $c_{\alpha}(x)$ in $L^{2}(\Omega)$ such that

$$
\frac{1}{\epsilon}\left(u_{\alpha}^{\epsilon}-\frac{1}{K} \sum_{\beta=1}^{K} u_{\beta}^{\epsilon}\right) \rightarrow \tilde{u}_{\alpha}^{1}(x, y)-\frac{1}{K} \sum_{\beta=1}^{K} \tilde{u}_{\beta}^{1}(x, y)+c_{\alpha}(x)
$$

in the sense of two-scale convergence. Remark that, since the sum over $\alpha$ of the left hand sides of (46) is zero, the functions $c_{\alpha}$ satisfy

$$
\sum_{\alpha=1}^{K} c_{\alpha}(x)=0 .
$$

Eventually, defining $u^{1}(x, y) \in L^{2}(\Omega ; \mathcal{H}(Y))$ by its components

$$
u_{\alpha}^{1}(x, y)=\tilde{u}_{\alpha}^{1}(x, y)+c_{\alpha}(x) \quad \forall \alpha \in\{1, . ., K\},
$$

we easily check that (46) implies the desired convergences (45).

\section{$5 \quad$ Homogenization}

This section is devoted to the proof of the homogenization Theorem 3.10. As in the previous section, $u^{\epsilon}=S_{\epsilon}\left(f^{\epsilon}\right)$ denotes the unique solution of (40) with $f^{\epsilon}$ a bounded sequence in $L^{2}(\Omega)^{K}$. We consider the subsequence for which Proposition 4.6 has established the existence of two-scale limits $u^{0}(x) \mathbb{I}$ and $u^{1}(x, y)$. Our goal is to characterize these limits as the solutions of some homogenized problems. If these solutions are unique, we shall conclude that the whole sequence $u^{\epsilon}$ converges, and not merely a subsequence. Let us first show that $u^{1}$ is uniquely determined by $u^{0}$.

Proposition 5.1 Let $u^{\epsilon}$ be the unique solution of system (40), and let $u^{0}(x) \mathbb{I}$ and $u^{1}(x, y)$ be its two-scale limits for a converging subsequence (see Proposition 4.6). Then $u^{1}(x, y)$ is a solution in $L^{2}(\Omega ; \mathcal{H}(Y))$ of the following system

$$
\left\{\begin{array}{l}
-\operatorname{div}_{y}\left(D(y) \nabla_{y} u^{1}(x, y)\right)+Q\left(u^{1}(x, y)\right)= \\
\quad \operatorname{div}_{y}\left(D(y) \mathbb{I} \otimes \nabla_{x} u^{0}(x)\right)-J(y) \mathbb{I} \otimes \nabla_{x} u^{0}(x) \quad \text { in } Y \\
y \rightarrow u^{1}(x, y) \quad \text { Y-periodic, a.e. } x \in \Omega .
\end{array}\right.
$$

where $Q$ is defined by $Q(u)=J(y) \nabla_{y} u+\tilde{Q}(y) u, J$ and $\tilde{Q}$ being introduced in (35), (34). 
Proof. For a smooth $Y$-periodic test function $\phi(x, y)$ (a vector with $K$ components), multiplying (40) by $\epsilon \phi(x, x / \epsilon)$ and integrating by parts yields

$$
\begin{gathered}
\int_{\Omega} D\left(\frac{x}{\epsilon}\right) \nabla u^{\epsilon} \cdot\left(\epsilon \nabla_{x} \phi\left(x, \frac{x}{\epsilon}\right)+\nabla_{y} \phi\left(x, \frac{x}{\epsilon}\right)\right) d x+\frac{1}{\epsilon} \int_{\Omega} Q^{\epsilon}\left(u^{\epsilon}\right) \cdot \phi\left(x, \frac{x}{\epsilon}\right) d x \\
=\epsilon \int_{\Omega} f^{\epsilon}(x) \cdot \phi\left(x, \frac{x}{\epsilon}\right) d x
\end{gathered}
$$

Since $f^{\epsilon}$ and $\nabla u^{\epsilon}$ are uniformly bounded in $L^{2}(\Omega)$, the right hand side and the first term of the left hand side in (48) vanishes as $\epsilon$ goes to zero. By application of Proposition 4.6 we can pass to the two-scale limit in the second term of the left hand side in (48)

$$
\begin{aligned}
\lim _{\epsilon \rightarrow 0} \int_{\Omega} D\left(\frac{x}{\epsilon}\right) \nabla u^{\epsilon} \cdot \nabla_{y} \phi\left(x, \frac{x}{\epsilon}\right) d x= \\
\quad \int_{\Omega} \int_{Y} D(y)\left(\mathbb{I} \otimes \nabla_{x} u^{0}(x)+\nabla_{y} u^{1}(x, y)\right) \cdot \nabla_{y} \phi(x, y) d x d y .
\end{aligned}
$$

The last term in (48) involves $Q^{\epsilon}\left(u^{\epsilon}\right)=\epsilon J(x / \epsilon) \nabla u^{\epsilon}+\tilde{Q}(x / \epsilon) u^{\epsilon}$. Clearly, by its definition (34), $J(y)$ is a $Y$-periodic function and we have

$$
\begin{aligned}
\lim _{\epsilon \rightarrow 0} & \int_{\Omega} J\left(\frac{x}{\epsilon}\right) \nabla u^{\epsilon} \cdot \phi\left(x, \frac{x}{\epsilon}\right) d x= \\
& \int_{\Omega} \int_{Y} J(y)\left(\mathbb{I} \otimes \nabla_{x} u^{0}(x)+\nabla_{y} u^{1}(x, y)\right) \cdot \phi(x, y) d x d y .
\end{aligned}
$$

On the other hand, by its definition (35), the matrix $\tilde{Q}$ satisfies $\tilde{Q}(y) \mathbb{I}=0$. Thus

$$
\frac{1}{\epsilon} \int_{\Omega} \tilde{Q}\left(\frac{x}{\epsilon}\right) u^{\epsilon} \cdot \phi\left(x, \frac{x}{\epsilon}\right) d x=\int_{\Omega} \tilde{Q}\left(\frac{x}{\epsilon}\right)\left(\frac{u^{\epsilon}-\mathbb{I}\left(u^{\epsilon}\right)}{\epsilon}\right) \cdot \phi\left(x, \frac{x}{\epsilon}\right) d x .
$$

By the $Y$-periodicity of $\tilde{Q}$ and the convergence result (45) of Proposition 4.6, we obtain

$$
\begin{aligned}
\lim _{\epsilon \rightarrow 0} \int_{\Omega} \tilde{Q}\left(\frac{x}{\epsilon}\right)\left(\frac{u^{\epsilon}-\mathbb{I}\left(u^{\epsilon}\right)}{\epsilon}\right) \cdot \phi\left(x, \frac{x}{\epsilon}\right) d x \\
\quad=\int_{\Omega} \int_{Y} \tilde{Q}(y)\left(u^{1}(x, y)-\mathbb{I}\left(u^{1}\right)(x, y)\right) \cdot \phi(x, y) d x d y \\
\quad=\int_{\Omega} \int_{Y} \tilde{Q}(y) u^{1}(x, y) \cdot \phi(x, y) d x d y
\end{aligned}
$$

because $\tilde{Q}(y) \mathbb{I}=0$. Summing up the above limits, we obtain the weak form of (47). 
From Proposition 5.1 we know that $u^{1}(x, y)$ is a solution of equation (47). However, at this point, it is not clear whether (47) admits a unique solution for any right hand side. In other words, depending on its solvability, equation (47) will either deliver the value of $u^{1}$ in terms of $\nabla_{x} u^{0}$, or force $\nabla_{x} u^{0}$ to take some precise values. It is the purpose of the following Lemma to give a Fredholm alternative for (47).

Lemma 5.2 Let $F \in L_{\#}^{2}(Y)^{K}$ with components $\left(F_{\alpha}\right)_{1 \leq \alpha \leq K}$. Let $\mathcal{H}(Y)$ be the Hilbert space defined by (44), i.e. $\mathcal{H}(Y)=H_{\#}^{1}(Y)^{K} /(\mathbb{R} \times \mathbb{I})$. There exists a unique solution in $\mathcal{H}(Y)$ of

$$
\left\{\begin{array}{l}
-\operatorname{div}(D(y) \nabla w)+Q(w)=F \\
y \rightarrow w(y) \quad Y-\text { periodic }
\end{array}\right.
$$

if and only if $\sum_{\alpha=1}^{K} \int_{Y} F_{\alpha}(y) d y=0$.

Proof. Let us first check that, if $\sum_{\alpha=1}^{K} \int_{Y} F_{\alpha} \neq 0$, there exists no solution of (49) in $H_{\#}^{1}(Y)^{K}$. Integrating the left hand side of (49), by periodicity we obtain that

$$
\int_{Y} \operatorname{div}(D(y) \nabla w) d y=0 .
$$

Furthermore, (37) implies that

$$
\int_{Y} J_{\alpha} \nabla w_{\alpha} d y=\sum_{\beta=1}^{K} \int_{Y}\left(\tilde{Q}_{\alpha, \beta} w_{\alpha}-\tilde{Q}_{\beta, \alpha} w_{\alpha}\right),
$$

and hence, taking into account $\sum_{\beta=1}^{K} \tilde{Q}_{\alpha, \beta}=0$,

$$
\sum_{\alpha=1}^{K} \int_{Y}\left(J_{\alpha} \nabla w_{\alpha}+\sum_{\beta=1}^{K} \tilde{Q}_{\alpha, \beta} w_{\beta}\right) d y=\sum_{\alpha=1}^{K} \sum_{\beta=1}^{K} \int_{Y} \tilde{Q}_{\alpha, \beta} w_{\alpha} d y=0
$$

Therefore, $\sum_{\alpha=1}^{K} \int_{Y} F_{\alpha}=0$ is a necessary condition of existence of solution. Assuming it is now satisfied, we check the assumptions of the Lax-Milgram theorem for the variational formulation of $(49)$ in $\mathcal{H}(Y)$. The bilinear form is coercive since

$$
\int_{Y} D(y) \nabla w \cdot \nabla w d y+\int_{Y} Q(w) \cdot w d y \geq C\left(\begin{array}{l}
\sum_{\alpha=1}^{K} \int_{Y}\left|\nabla w_{\alpha}\right|^{2} d y \\
+\sum_{\alpha, \beta=1}^{K} \int_{Y}\left(w_{\alpha}-w_{\beta}\right)^{2} d y
\end{array}\right),
$$

where the right hand side defines a norm on $\mathcal{H}(Y)$ (its kernel in $H_{\#}^{1}(Y)^{K}$ is the one dimensional subspace span by $\mathbb{I}$ ). On the other hand, the compatibility 
condition on $F$ implies that $F$ is orthogonal to $\mathbb{I}$ which clearly implies that the linear form $\phi \rightarrow \int_{Y} F \cdot \phi d y$ is continuous on $\mathcal{H}(Y)$. We now check the continuity of the bilinear form where the only difficulty is to estimate the term $\int_{Y} Q(w) \cdot v d y$. Let us first remark that the preceding computation has shown that

$$
\int_{Y} Q(w) \cdot \mathbb{I} d y=0
$$

Therefore, together with the fact that $Q(w+\lambda \mathbb{I})=Q(w)$ for any $\lambda \in \mathbb{R}$, it leads to the identity

$$
\int_{Y} Q(w) \cdot v d y=\int_{Y} Q\left(w-\mathbb{I}\left(\int_{Y} w\right)\right) \cdot\left(v-\mathbb{I}\left(\int_{Y} v\right)\right) d y
$$

for any $w, v$ in $\mathcal{H}(Y)$. Recall that in Proposition 3.7 we proved that the operator $Q^{\epsilon}$ is continuous from $H_{0}^{1}(\Omega)^{K}$ into $H^{-1}(\Omega)^{K}$. Since $Q^{\epsilon}$ and $Q$ are identical, up to a scaling of order $\epsilon$, a similar argument shows the existence of a constant $C$ such that, for any $w, v \in \mathcal{H}(Y)$,

$$
\left|\int_{Y} Q(w) \cdot v d y\right| \leq C\|w\|_{H_{\#}^{1}(Y)^{K}}\|v\|_{H_{\#}^{1}(Y)^{K}} .
$$

Using (50) leads to

$$
\left|\int_{Y} Q(w) \cdot v d y\right| \leq C\left\|w-\mathbb{I}\left(\int_{Y} w\right)\right\|_{H_{\#}^{1}(Y)^{K}}\left\|v-\mathbb{I}\left(\int_{Y} v\right)\right\|_{H_{\#}^{1}(Y)^{K}},
$$

where $\left\|w-\mathbb{I}\left(\int_{Y} w\right)\right\|_{H_{\#}^{1}(Y)^{K}}$ is just the norm in $\mathcal{H}(Y)$. Finally, application of the the Lax-Milgram theorem in $\mathcal{H}(Y)$ yields the existence and uniqueness of a solution for (49).

Proposition 5.3 Let $u^{0}(x) \in H_{0}^{1}(\Omega)$ and $u^{1}(x, y) \in L^{2}(\Omega ; \mathcal{H}(Y))$ be the limits satisfying system (47). Then, if

$$
\sum_{\alpha=1}^{K} \int_{Y} J_{\alpha}(y) d y \neq 0,
$$

necessarily $u^{0}(x)=0$ in $\Omega$. Conversely, if

$$
\sum_{\alpha=1}^{K} \int_{Y} J_{\alpha}(y) d y=0,
$$

then $u^{1}(x, y)$ is explicitly given by its components

$$
u_{\alpha}^{1}(x, y)=\sum_{i=1}^{N} \theta_{i, \alpha}(y) \frac{\partial u^{0}}{\partial x_{i}}(x),
$$


where, for $1 \leq i \leq N, \theta_{i}=\left(\theta_{i, \alpha}\right)_{1 \leq \alpha \leq K}$ is the unique solution in $\mathcal{H}(Y)$ of

$$
\left\{\begin{array}{l}
-\operatorname{div}\left(D(y) \nabla\left(\theta_{i}(y)+y_{i} \mathbb{I}\right)\right)+Q\left(\theta_{i}(y)+y_{i} \mathbb{I}\right)=0 \\
y \rightarrow \theta_{i}(y) \quad Y \text {-periodic. }
\end{array}\right.
$$

Remark 5.4 The condition (51) is nothing but our previous symmetry condition (18). Here, it appears as a Fredholm alternative for the cell problem (49).

Proof. According to Lemma 5.2, the Fredholm alternative for equation (47) is

$$
\left(\sum_{\alpha=1}^{K} \int_{Y} J_{\alpha}(y) d y\right) \cdot \nabla u^{0}(x)=0 \text { a.e. } x \in \Omega .
$$

If (51) is not satisfied, it implies that, at least, one component of $\nabla u^{0}$ vanishes throughout $\Omega$. Because of the homogeneous Dirichlet boundary condition, it yields that $u^{0}(x)=0$ in $\Omega$. If $(51)$ is satisfied, then Lemma 5.2 states that $(47)$ admits a unique solution $u^{1}$. By linearity it is easily seen to coincide with the prescribed combination of the functions $\theta_{i}$.

Remark 5.5 The adjoint of $Q$, noted $Q^{*}$ is given by

$$
Q^{*}(u)=-J(y) \dot{\nabla} u-\operatorname{div} J(y) \cdot u+\tilde{Q}^{*}(y) u
$$

Where $\tilde{Q}^{*}$ is the transposed matrix of $\tilde{Q}$. Using identity (37) we rewrite $Q^{*}$ under the following form:

$$
Q^{*}(u)=-J(y) \nabla u+\tilde{Q}^{a}(y) u
$$

Where $\tilde{Q}^{a}$ has the same off-diagonal entries than $\tilde{Q}^{*}$, and has diagonal entries such that, for all $1 \leq \alpha \leq K$,

$$
\sum_{\beta=1}^{K} \tilde{Q}_{\alpha, \beta}^{a}=0
$$

Both operators $Q$ and $Q^{*}$ have the same kernel, span by $\mathbb{I}$. Thus the existence and uniqueness result of Lemma 5.2 extends easily to the same equation with $Q$ replaced by $Q^{*}$. We therefore introduce adjoint functions $\theta_{i}^{*}(y), 1 \leq i \leq N$, defined as the unique solution in $\mathcal{H}(Y)$ of

$$
\left\{\begin{array}{l}
-\operatorname{div}\left(D(y) \nabla\left(\theta_{i}^{*}(y)+y_{i} \mathbb{I}\right)\right)+Q^{*}\left(\theta_{i}^{*}(y)+y_{i} \mathbb{I}\right)=0 \\
y \rightarrow \theta_{i}^{*}(y) \quad Y \text {-periodic. }
\end{array}\right.
$$

Proof of proposition 3.10. The principle of this proof is in the spirit of the so-called energy method, introduced by Tartar (see e.g. [11], [25]). We use an oscillating test function that has the same structure than the two-scale limit of 
$u^{\epsilon}$. Let $\varphi(x)$ be a smooth scalar function with compact support in $\Omega$. Define the vector-valued function $\phi(x)=\varphi(x) \mathbb{I}$ and $\phi^{1}(x, y)$ by its $K$ components

$$
\phi_{\alpha}^{1}(x, y)=\sum_{i=1}^{N} \theta_{i, \alpha}^{*}(y) \frac{\partial \varphi}{\partial x_{i}}(x) .
$$

By rescaling (53), $\theta_{i, \alpha}^{*}\left(\frac{x}{\epsilon}\right)$ satisfies in $\mathbb{R}^{N}$ the following equation

$$
\begin{aligned}
&-\epsilon \operatorname{div}\left(D\left(\frac{x}{\epsilon}\right) \nabla\left(\epsilon \theta_{i}^{*}\left(\frac{x}{\epsilon}\right)+x_{i}\right)\right) \\
&-J\left(\frac{x}{\epsilon}\right) \nabla\left(\epsilon \theta_{i}^{*}\left(\frac{x}{\epsilon}\right)+x_{i}\right)+\tilde{Q}^{a}\left(\frac{x}{\epsilon}\right) \theta_{i}^{*}\left(\frac{x}{\epsilon}\right)=0 .
\end{aligned}
$$

Multiplying equation (40), satisfied by $u^{\epsilon}$, by $\phi(x)+\epsilon \phi^{1}\left(x, \frac{x}{\epsilon}\right)$ and integrating by parts leads to

$$
\begin{aligned}
& \int_{\Omega} D\left(\frac{x}{\epsilon}\right) \nabla u^{\epsilon}(x) \cdot \nabla\left(\phi(x)+\epsilon \phi^{1}\left(x, \frac{x}{\epsilon}\right)\right) d x \\
& +\frac{1}{\epsilon^{2}} \int_{\Omega} Q^{\epsilon}\left(u^{\epsilon}\right)(x) \cdot\left(\phi(x)+\epsilon \phi^{1}\left(x, \frac{x}{\epsilon}\right)\right) d x=\int_{\Omega} f^{\epsilon}(x) \cdot\left(\phi(x)+\epsilon \phi^{1}\left(x, \frac{x}{\epsilon}\right)\right) d x .
\end{aligned}
$$

The right hand side converges to

$$
\int_{\Omega} f(x) \cdot \phi(x) d x=\int_{\Omega}\left(\sum_{\alpha=1}^{K} f_{\alpha}(x)\right) \varphi(x) d x
$$

as $\epsilon$ goes to zero, and

$$
\lim _{\epsilon \rightarrow 0} \epsilon \int_{\Omega} D\left(\frac{x}{\epsilon}\right) \nabla u^{\epsilon}(x) \cdot \nabla_{x} \phi^{1}\left(x, \frac{x}{\epsilon}\right) d x=0 .
$$

Therefore the above equation writes

$$
\begin{aligned}
& \int_{\Omega} D\left(\frac{x}{\epsilon}\right) \nabla u^{\epsilon}(x) \cdot\left(\nabla \phi(x)+\nabla_{y} \phi^{1}\left(x, \frac{x}{\epsilon}\right)\right) d x \\
& +\frac{1}{\epsilon^{2}} \int_{\Omega} u^{\epsilon}(x) \cdot Q^{\epsilon *}\left(\phi(x)+\epsilon \phi^{1}\left(x, \frac{x}{\epsilon}\right)\right) d x=\int_{\Omega} f(x) \cdot \phi(x) d x+r(\epsilon),
\end{aligned}
$$

where $r(\epsilon)$ is a bounded quantity going to zero with $\epsilon$. Using the definition of $Q^{*}$, i.e. $Q^{*}(u)=-J(y) \nabla u+\tilde{Q}^{a}(y) u$ and definition (54) of $\phi^{1}$, the left-hand-side 
of (56) becomes

$$
\begin{aligned}
& \sum_{i=1}^{N} \sum_{\alpha=1}^{K} \int_{\Omega} D_{\alpha}\left(\frac{x}{\epsilon}\right) \nabla u_{\alpha}^{\epsilon} \cdot \frac{\partial \varphi}{\partial x_{i}}\left(\nabla x_{i}+\left(\nabla_{y} \theta_{i, \alpha}^{*}\right)\left(\frac{x}{\epsilon}\right)\right) d x \\
& -\frac{1}{\epsilon} \sum_{i=1}^{N} \sum_{\alpha=1}^{K} \int_{\Omega} u_{\alpha}^{\epsilon} J_{\alpha}\left(\frac{x}{\epsilon}\right) \cdot \frac{\partial \varphi}{\partial x_{i}}\left(\nabla x_{i}+\left(\nabla_{y} \theta_{i, \alpha}^{*}\right)\left(\frac{x}{\epsilon}\right)\right) d x \\
& -\sum_{i=1}^{N} \sum_{\alpha=1}^{K} \int_{\Omega} u_{\alpha}^{\epsilon} J_{\alpha}\left(\frac{x}{\epsilon}\right) \cdot \nabla \frac{\partial \varphi}{\partial x_{i}} \theta_{i, \alpha}^{*}\left(\frac{x}{\epsilon}\right) d x \\
& +\frac{1}{\epsilon^{2}} \int_{\Omega} \tilde{Q}^{a}\left(\frac{x}{\epsilon}\right) \mathbb{I} \cdot \varphi u^{\epsilon} d x+\frac{1}{\epsilon} \sum_{i=1}^{N} \int_{\Omega} \tilde{Q}^{a}\left(\frac{x}{\epsilon}\right) \theta_{i}^{*}\left(\frac{x}{\epsilon}\right) \cdot \frac{\partial \varphi}{\partial x_{i}} u^{\epsilon} d x .
\end{aligned}
$$

Remark that $\int_{\Omega} \tilde{Q}^{a}\left(\frac{x}{\epsilon}\right) \mathbb{I} \cdot \varphi u^{\epsilon} d x=0$, since $\tilde{Q}^{a} \mathbb{I}=0$. On the other hand, multiplying equation (55), satisfied by $\theta_{i}^{*}$, by $\frac{\partial \varphi}{\partial x_{i}} u^{\epsilon}$, we obtain

$$
\begin{aligned}
& \sum_{\alpha=1}^{K} \int_{\Omega} D_{\alpha}\left(\frac{x}{\epsilon}\right)\left(\nabla x_{i}+\left(\nabla_{y} \theta_{i, \alpha}^{*}\right)\left(\frac{x}{\epsilon}\right)\right) \cdot\left(\frac{\partial \varphi}{\partial x_{i}} \nabla u_{\alpha}^{\epsilon}+u_{\alpha}^{\epsilon} \nabla\left(\frac{\partial \varphi}{\partial x_{i}}\right)\right) d x \\
& -\frac{1}{\epsilon} \sum_{\alpha=1}^{K} \int_{\Omega} J_{\alpha}\left(\frac{x}{\epsilon}\right)\left(\nabla x_{i}+\left(\nabla_{y} \theta_{i, \alpha}^{*}\right)\left(\frac{x}{\epsilon}\right)\right) \cdot \frac{\partial \varphi}{\partial x_{i}} u_{\alpha}^{\epsilon} d x \\
& +\frac{1}{\epsilon} \int_{\Omega} \tilde{Q}^{a}\left(\frac{x}{\epsilon}\right) \theta_{i}^{*}\left(\frac{x}{\epsilon}\right) \cdot \frac{\partial \varphi}{\partial x_{i}} u_{\alpha}^{\epsilon} d x=0 .
\end{aligned}
$$

Thus, using (57) in (56) and subtracting to it equation (58) yields

$$
\begin{aligned}
& -\sum_{i=1}^{N} \sum_{\alpha=1}^{K} \int_{\Omega} D_{\alpha}\left(\frac{x}{\epsilon}\right)\left(\nabla x_{i}+\left(\nabla_{y} \theta_{i, \alpha}^{*}\right)\left(\frac{x}{\epsilon}\right)\right) \cdot u_{\alpha}^{\epsilon} \nabla\left(\frac{\partial \varphi}{\partial x_{i}}\right) d x \\
& \quad-\sum_{i=1}^{N} \sum_{\alpha=1}^{K} \int_{\Omega} J_{\alpha}\left(\frac{x}{\epsilon}\right) \cdot \nabla\left(\frac{\partial \varphi}{\partial x_{i}}\right) \theta_{i, \alpha}^{*}\left(\frac{x}{\epsilon}\right) u_{\alpha}^{\epsilon} d x=\int_{\Omega}\left(\sum_{\alpha=1}^{K} f_{\alpha}\right) \varphi d x+r(\epsilon) .
\end{aligned}
$$

All terms in the left-hand-side of this last expression are products of $u^{\epsilon}$, which converges strongly in $L^{2}(\Omega)$ towards $u_{0}(x) \mathbb{I}$, against periodically oscillating functions that converge weakly in $L^{2}(\Omega)$. Taking the limit as $\epsilon$ goes to zero, 
and after an integration by parts, it yields

$$
\begin{aligned}
& \sum_{i, j=1}^{N} \int_{\Omega} \frac{\partial u^{0}}{\partial x_{j}}(x) \frac{\partial \varphi}{\partial x_{i}}(x) \sum_{\alpha=1}^{K} \int_{Y} \theta_{i, \alpha}^{*}(y) J_{\alpha}(y) \cdot \nabla y_{j} d y d x+ \\
& \sum_{i, j=1}^{N} \int_{\Omega} \frac{\partial u^{0}}{\partial x_{j}}(x) \frac{\partial \varphi}{\partial x_{i}}(x) \sum_{\alpha=1}^{K} \int_{Y} D_{\alpha}(y) \nabla\left(y_{i}+\theta_{i, \alpha}^{*}(y)\right) \cdot \nabla y_{j} d y d x= \\
& \int_{\Omega} \sum_{\alpha=1}^{K} f_{\alpha}(x) \varphi(x) d x .
\end{aligned}
$$

Introducing a matrix $\tilde{D}$ defined by its entries

$$
\tilde{D}_{i, j}=\sum_{\alpha=1}^{K} \int_{Y}\left(D_{\alpha}(y) \nabla\left(y_{i}+\theta_{i, \alpha}^{*}(y)\right) \cdot \nabla y_{j}+\theta_{i, \alpha}^{*}(y) J_{\alpha}(y) \cdot \nabla y_{j}\right) d y
$$

equation (59) is just a variational formulation of

$$
\left\{\begin{array}{l}
-\operatorname{div}\left(\tilde{D} \nabla u^{0}(x)\right)=\sum_{\alpha=1}^{K} f_{\alpha}(x) \text { in } \Omega, \\
u^{0} \in H_{0}^{1}(\Omega)
\end{array}\right.
$$

In Proposition 5.6 we shall assert that the constant matrix $\tilde{D}$ has a positive definite symmetrical part. Therefore, there exists a unique solution $u^{0}$, which finishes the proof of Proposition 3.10.

The homogenized matrix $\tilde{D}$, introduced in the above proof, is not, at first look, the one given in the statement of Theorem 3.2 and denoted by $\bar{D}$. In particular, the matrix $\tilde{D}$ is not symmetric as is $\bar{D}$. This is not a problem since only the symmetric part of $\tilde{D}$ plays a role in the homogenized diffusion equation (61). The purpose of the next Proposition is to show that the symmetric part of $\tilde{D}$ is positive definite, which implies that the homogenized diffusion equation (61) is well-posed. Then, Proposition 5.7 shows that this symmetric part of $\tilde{D}$ coincides with $\bar{D}$.

Proposition 5.6 Let $\tilde{D}^{s}$ denote the symmetric part of $\tilde{D}$, defined by (60). An equivalent formula for $\tilde{D}^{s}$ is

$$
\begin{aligned}
\tilde{D}_{i, j}^{s}= & \sum_{\alpha=1}^{K} \int_{Y} D_{\alpha}(y) \nabla\left(y_{i}+\theta_{i, \alpha}^{*}(y)\right) \cdot \nabla\left(y_{j}+\theta_{j, \alpha}^{*}(y)\right) d y \\
& -\frac{1}{2} \sum_{\alpha, \beta=1}^{K} \int_{Y} \tilde{Q}_{\alpha, \beta}(y)\left(\theta_{i, \alpha}^{*}(y)-\theta_{i, \beta}^{*}(y)\right) \cdot\left(\theta_{j, \alpha}^{*}(y)-\theta_{j, \beta}^{*}(y)\right) d y .
\end{aligned}
$$

In particular, $\tilde{D}^{s}$ is positive definite. 
Proof. Multiplying definition (53) of $\theta_{i}^{*}$ by $\theta_{j}^{*}$ and integrating by parts leads to

$$
\begin{aligned}
& \sum_{\alpha=1}^{K} \int_{Y} D_{\alpha} \nabla\left(y_{i}+\theta_{i, \alpha}^{*}\right) \cdot \nabla \theta_{j, \alpha}^{*} d y \\
& -\sum_{\alpha=1}^{K} \int_{Y} J_{\alpha} \nabla\left(y_{i}+\theta_{i, \alpha}^{*}\right) \cdot \theta_{j, \alpha}^{*} d y+\int_{Y} \tilde{Q}^{a} \theta_{i}^{*} \cdot \theta_{j}^{*} d y=0 .
\end{aligned}
$$

Therefore, (60) is equivalent to

$$
\begin{aligned}
\tilde{D}_{i, j}= & \sum_{\alpha=1}^{K} \int_{Y} D_{\alpha} \nabla\left(y_{i}+\theta_{i, \alpha}^{*}\right) \cdot \nabla\left(y_{j}+\theta_{j, \alpha}^{*}\right) d y \\
& +\int_{Y} Q^{*}\left(\theta_{i}^{*}\right) \cdot \theta_{j}^{*} d y+\sum_{\alpha=1}^{K} \int_{Y}\left(J_{\alpha} \nabla y_{j} \cdot \theta_{i, \alpha}^{*}-J_{\alpha} \nabla y_{i} \cdot \theta_{j, \alpha}^{*}\right) d y,
\end{aligned}
$$

and its symmetrical part is

$$
\begin{aligned}
\tilde{D}_{i, j}^{s}= & \sum_{\alpha=1}^{K} \int_{Y} D_{\alpha} \nabla\left(y_{i}+\theta_{i, \alpha}^{*}\right) \cdot \nabla\left(y_{j}+\theta_{j, \alpha}^{*}\right) d y \\
& +\frac{1}{2} \int_{Y}\left(Q^{*}+Q\right)\left(\theta_{i}^{*}\right) \cdot \theta_{j}^{*} d y
\end{aligned}
$$

We proved in proposition 3.7 that

$$
\int_{Y} Q(w) \cdot w d y=-\frac{1}{2} \sum_{\alpha, \beta=1}^{K} \int_{Y} \tilde{Q}_{\alpha, \beta}\left(w_{\alpha}-w_{\beta}\right)^{2} d y
$$

Thus, taking the corresponding symmetric bilinear form we obtain

$$
\frac{1}{2} \int_{Y}\left(Q^{*}+Q\right)\left(\theta_{i}^{*}\right) \cdot \theta_{j}^{*} d y=-\frac{1}{2} \sum_{\alpha, \beta=1}^{K} \int_{Y} \tilde{Q}_{\alpha, \beta}\left(\theta_{i, \alpha}^{*}-\theta_{i, \beta}^{*}\right) \cdot\left(\theta_{j, \alpha}^{*}-\theta_{j, \beta}^{*}\right) d y,
$$

and formula (62) follows. Regarding the coercivity of $\tilde{D}^{s}$, we have, for all $\xi \in \mathbb{R}^{N}$,

$$
\begin{aligned}
\sum_{i, j=1}^{N} \tilde{D}_{i j}^{s} \xi_{i} \xi_{j} \geq & \sum_{\alpha=1}^{K} \sum_{i, j=1}^{N} \int_{Y} D_{\alpha} \nabla\left(y_{i}+\theta_{i, \alpha}^{*}\right) \nabla\left(y_{j}+\theta_{j, \alpha}^{*}\right) \xi_{i} \xi_{j} d y \\
& -\frac{1}{2} \sum_{\alpha, \beta=1}^{K} \sum_{i, j=1}^{N} \int_{Y} \tilde{Q}_{\alpha, \beta}\left(\theta_{i, \alpha}^{*}-\theta_{i, \beta}^{*}\right)\left(\theta_{j, \alpha}^{*}-\theta_{j, \beta}^{*}\right) \xi_{i} \xi_{j} d y \\
\geq & C \sum_{\alpha=1}^{K} \int_{Y}\left|\nabla\left(\sum_{i=1}^{N} \xi_{i}\left(y_{i}-\theta_{i, \alpha}^{*}\right)\right)\right|^{2} d y \\
& -\frac{1}{2} \sum_{\alpha, \beta=1}^{K} \int_{Y} \tilde{Q}_{\alpha, \beta}\left(\sum_{i=1}^{N}\left(\theta_{i, \alpha}^{*}-\theta_{i, \beta}^{*}\right) \xi_{i}\right)^{2} d y
\end{aligned}
$$


where $C>0$ is the coercivity constant of $D_{\alpha}$, for all $\alpha$. Since $\tilde{Q}_{\alpha, \beta} \leq 0$ for all $\alpha \neq \beta$, the second term is also positive, which proves that $\tilde{D}^{s}$ is positive definite.

Proposition 5.7 The symmetrical part of $\tilde{D}$ coincide with $\bar{D}$, i.e.

$$
\begin{array}{r}
\tilde{D}_{i, j}^{s}=\bar{D}_{i, j}=\sum_{\alpha=1}^{K} \int_{Y} D_{\alpha}(y) \nabla\left(y_{i}+\theta_{i, \alpha}(y)\right) \cdot \nabla\left(y_{j}+\theta_{j, \alpha}(y)\right) d y \\
\quad-\frac{1}{2} \sum_{\alpha, \beta=1}^{K} \int_{Y} \tilde{Q}_{\alpha, \beta}(y)\left(\theta_{i, \alpha}(y)-\theta_{i, \beta}(y)\right) \cdot\left(\theta_{j, \alpha}(y)-\theta_{j, \beta}(y)\right) d y .
\end{array}
$$

Proof. From (60) we get

$$
\begin{aligned}
\tilde{D}_{i, j} & =\sum_{\alpha=1}^{K} \int_{Y} D_{\alpha} \nabla y_{i} \cdot \nabla\left(\theta_{j, \alpha}+y_{j}\right) d y+\sum_{\alpha=1}^{K} \int_{Y} J_{\alpha} \nabla y_{j} \cdot \theta_{i, \alpha}^{*} d y \\
& +\sum_{\alpha=1}^{K} \int_{Y} D_{\alpha} \nabla \theta_{i, \alpha}^{*} \cdot \nabla\left(\theta_{j, \alpha}+y_{j}\right) d y-\sum_{\alpha=1}^{K} \int_{Y} D_{\alpha} \nabla\left(\theta_{i, \alpha}^{*}+y_{i}\right) \cdot \nabla \theta_{j, \alpha} d y .
\end{aligned}
$$

The definitions (53) of $\theta_{j}^{*}$ and (52) of $\theta_{i}$ gives that

$$
\begin{aligned}
& \sum_{\alpha=1}^{K} \int_{Y} D_{\alpha} \nabla \theta_{i, \alpha}^{*} \cdot \nabla\left(\theta_{j, \alpha}+y_{j}\right) d y \\
& \quad-\sum_{\alpha=1}^{K} \int_{Y} D_{\alpha} \nabla\left(\theta_{i, \alpha}^{*}+y_{i}\right) \cdot \nabla \theta_{j, \alpha} d y \\
& =-\int_{Y} Q\left(\theta_{j}+y_{j}\right) \cdot \theta_{i}^{*} d y+\int_{Y} Q^{*}\left(\theta_{i}^{*}+y_{i}\right) \cdot \theta_{j} d y \\
& =-\sum_{\alpha=1}^{K} \int_{Y} J_{\alpha} \cdot \nabla y_{j} \theta_{i, \alpha}^{*} d y-\sum_{\alpha=1}^{K} \int_{Y} J_{\alpha} \cdot \nabla y_{i} \theta_{j, \alpha} d y .
\end{aligned}
$$

Thus, the homogenized matrix $\tilde{D}$ is also given by

$$
\tilde{D}_{i, j}=\sum_{\alpha=1}^{K} \int_{Y} D_{\alpha} \nabla y_{i} \cdot \nabla\left(\theta_{j, \alpha}+y_{j}\right) d y-\sum_{\alpha=1}^{K} \int_{Y} \theta_{j, \alpha} J_{\alpha} \cdot \nabla y_{i} d y .
$$

Now, formula (64) for $\tilde{D}$, expressed in terms of $\theta_{i}$, can be compared with formula (60), and arguing as in the proof of Proposition 5.6 leads to the desired formula $(63)$.

Remark 5.8 The functions $\left(\theta_{i}\right)_{1 \leq i \leq N}$ have been defined in two different ways. In Theorem 3.2 they are defined as the solutions of system (20), whereas in Proposition 5.3 they are solutions of system (52). Our notations are consistent in the sense that (5D) is just (2D), each line being multiplied by $\psi_{\alpha} \psi_{\alpha}^{*}$. 
Remark 5.9 As shown above, the homogenized diffusion matrix $\bar{D}$ can be defined, as in (21), in terms of corrector functions $\left(\theta_{i}\right)_{1 \leq i \leq N}$, or, as in (62), in terms of adjoint correctors $\left(\theta_{i}^{*}\right)_{1 \leq i \leq N}$. In fact, the introduction of adjoint correctors is not compulsory for obtaining the homogenized limit: the proof of Proposition 3.10 can also be done with test functions defined through direct correctors $\theta_{i, \alpha}$, even though the limit formula appears in a more complicated form.

The fact that we can characterize the homogenized matrix $\tilde{D}$ with either direct or adjoint correctors enlightens the meaning of the symmetry condition (18) we have assumed. Indeed, had we addressed the adjoint problem of (8), we would have obtained that, once factorized by the periodic eigenvector $\psi^{*}$, it converged to the very same eigenvalue problem. Therefore, the macroscopic behaviour of the direct and adjoint eigenvectors of problem (8) are asymptotically equal. The symmetry condition (18) implies that $\epsilon$-scale oscillations capture the non-adjointness of the problem.

\section{A corrector result}

In this section we show that, under the symmetry assumption (18), the so-called correctors $u^{1}(x, y)$ can actually improve the convergence result. In other words this justifies the $\epsilon$ order terms of the asymptotic expansion of $u^{\epsilon}$. To obtain this result we follow the approach in [1].

Theorem 6.1 Let $f^{\epsilon}$ be a sequence which converges weakly in $L^{2}(\Omega)^{K}$ to $f$ with components $\left(f_{\alpha}\right)_{1<\alpha \leq K}$. Let $u^{\epsilon}$ be defined as the unique solution in $H_{0}^{1}(\Omega)^{K}$ of (40). Let $u^{0}(x)$ be the unique solution in $H_{1}^{0}(\Omega)$ of $(61)$ and $u^{1}(x, y)$ be the unique solution in $L^{2}(\Omega ; \mathcal{H}(Y))$ of (47). Suppose that the symmetry condition (18) is observed, and $u^{0} \in H_{0}^{2}(\Omega)$. Then

$$
u^{\epsilon}-u^{0}(x) \mathbb{I}-\epsilon u^{1}\left(x, \frac{x}{\epsilon}\right) \rightarrow 0 \quad \text { strongly in } H_{0}^{1}(\Omega)
$$

Remark 6.2 This corrector result can be applied to the original eigenvalue problem (8). Indeed, Theorem 3.2 insures the convergence of $\epsilon^{-2}\left(\mu^{\epsilon}-\mu^{\infty}\right)$ in $\mathbb{R}$, and of $u^{\epsilon}$ in $L^{2}(\Omega)$ strong. Thus the right-hand-side of (27) satisfies the same hypothesis as $f^{\epsilon}$ does. In that particular case, $u^{0}$ is smooth, as a solution of a constant coefficient elliptic eigenvalue problem.

Proof. Let us first remark that $\nabla u_{\alpha}^{1}\left(x, \frac{x}{\epsilon}\right)$ belongs to $L^{2}(\Omega)^{N}$ for all $1 \leq \alpha \leq K$, because of the regularity of $u^{0}$. It is sufficient to prove that

$$
\lim _{\epsilon \rightarrow 0} \sum_{\alpha=1}^{K} \int_{\Omega} D_{\alpha}\left(\frac{x}{\epsilon}\right) \nabla \zeta_{\alpha}^{\epsilon} \cdot \nabla \zeta_{\alpha}^{\epsilon} d x=0
$$


with $\zeta_{\alpha}^{\epsilon}=u_{\alpha}^{\epsilon}-u^{0}(x)-\epsilon u_{\alpha}^{1}\left(x, \frac{x}{\epsilon}\right)$. Multiplying equation (40) by $u^{\epsilon}$, integrating by parts and using identity (38) yields

$$
\begin{gathered}
\sum_{\alpha=1}^{K} \int_{\Omega} D_{\alpha}\left(\frac{x}{\epsilon}\right) \nabla u_{\alpha}^{\epsilon} \cdot \nabla u_{\alpha}^{\epsilon} d x-\frac{1}{2 \epsilon^{2}} \sum_{\alpha, \beta=1}^{K} \int_{\Omega} \tilde{Q}_{\alpha, \beta}\left(\frac{x}{\epsilon}\right)\left(u_{\alpha}^{\epsilon}-u_{\beta}^{\epsilon}\right)^{2} d x \\
=\sum_{\alpha=1}^{K} \int_{\Omega} f_{\alpha}^{\epsilon} \cdot u_{\alpha}^{\epsilon} d x .
\end{gathered}
$$

Using the above identity in the expansion of $D\left(\frac{x}{\epsilon}\right) \nabla \zeta^{\epsilon} \cdot \nabla \zeta^{\epsilon}$ we obtain

$$
\begin{aligned}
& \sum_{\alpha=1}^{K} \int_{\Omega} D_{\alpha}\left(\frac{x}{\epsilon}\right) \nabla \zeta_{\alpha}^{\epsilon} \cdot \nabla \zeta_{\alpha}^{\epsilon} d x=\frac{1}{2 \epsilon^{2}} \sum_{\alpha, \beta=1}^{K} \int_{\Omega} \tilde{Q}_{\alpha, \beta}\left(\frac{x}{\epsilon}\right)\left(u_{\alpha}^{\epsilon}-u_{\beta}^{\epsilon}\right)^{2} d x \\
& +\sum_{\alpha=1}^{K} \int_{\Omega} D_{\alpha}\left(\frac{x}{\epsilon}\right) \nabla\left(u^{0}(x)+\epsilon u_{\alpha}^{1}\left(x, \frac{x}{\epsilon}\right)\right) \cdot \nabla\left(u^{0}(x)+\epsilon u_{\alpha}^{1}\left(x, \frac{x}{\epsilon}\right)\right) d x \\
& -2 \sum_{\alpha=1}^{K} \int_{\Omega} D_{\alpha}\left(\frac{x}{\epsilon}\right) \nabla u_{\alpha}^{\epsilon} \cdot \nabla\left(u^{0}(x)+\epsilon u_{\alpha}^{1}\left(x, \frac{x}{\epsilon}\right)\right) d x+\sum_{\alpha=1}^{K} \int_{\Omega} f_{\alpha}^{\epsilon} \cdot u_{\alpha}^{\epsilon} d x .
\end{aligned}
$$

The last three terms of the right-hand-side converges as $\epsilon$ goes to zero to their two-scale limits. The only difficulty lies in the first term of the right-handside, which is a product of two-scale weakly converging terms. We know from Proposition 1.6 of [1], that if $v_{0}(x, y)$ is the two scale limit of a sequence $v_{\epsilon}$ in $L^{2}(\Omega)$ we have

$$
\lim _{\epsilon \rightarrow 0}\left\|v_{\epsilon}\right\|_{L^{2}(\Omega)} \geq\left\|v_{0}\right\|_{L^{2}(\Omega \times Y)} .
$$

In Proposition 4.6 we established that the two-scale limit of $\frac{1}{\epsilon}\left(u^{\epsilon}-\mathbb{I}\left(u^{\epsilon}\right)\right)$ was $u^{1}(x, y)-\mathbb{I}\left(u^{1}\right)$. It implies that $\tilde{Q}_{\alpha, \beta}(y)\left(u_{\alpha}^{1}(x, y)-u_{\beta}^{1}(x, y)\right)$ is the two scale limit of $\frac{1}{\epsilon} \tilde{Q}_{\alpha, \beta}\left(\frac{x}{\epsilon}\right)\left(u_{\alpha}^{\epsilon}-u_{\beta}^{\epsilon}\right)$, for all $1 \leq \alpha, \beta \leq K$. Keeping in mind that $\tilde{Q}_{\alpha, \beta} \leq 0$ if $\alpha \neq \beta$, the above inequality implies that

$\lim _{\epsilon \rightarrow 0} \frac{1}{\epsilon^{2}} \int_{\Omega} \tilde{Q}_{\alpha, \beta}\left(\frac{x}{\epsilon}\right)\left(u_{\alpha}^{\epsilon}-u_{\beta}^{\epsilon}\right)^{2} d x \leq \int_{\Omega} \int_{Y} \tilde{Q}_{\alpha, \beta}(y)\left(u_{\alpha}^{1}(x, y)-u_{\beta}^{1}(x, y)\right)^{2} d y d x$. 
Thus, taking the limit of all quantities in (65) as $\epsilon$ goes to zero,

$$
\begin{aligned}
& \lim _{\epsilon \rightarrow 0} \sum_{\alpha=1}^{K} \int_{\Omega} D_{\alpha}\left(\frac{x}{\epsilon}\right) \nabla \zeta_{\alpha}^{\epsilon} \cdot \nabla \zeta_{\alpha}^{\epsilon} d x \\
& \leq \frac{1}{2} \sum_{\alpha, \beta=1}^{K} \int_{Y} \tilde{Q}_{\alpha, \beta}(y)\left(u_{\alpha}^{1}(x, y)-u_{\beta}^{1}(x, y)\right)^{2} d x d y \\
& -\sum_{\alpha=1}^{K} \int_{\Omega} \int_{Y} D_{\alpha}(y)\left(\nabla u^{0}(x)-\nabla_{y} u_{\alpha}^{1}(x, y)\right) \cdot\left(\nabla u^{0}(x)-\nabla_{y} u_{\alpha}^{1}(x, y)\right) d x d y \\
& +\sum_{\alpha=1}^{K} \int_{\Omega} f_{\alpha}(x) u_{0}(x) d x
\end{aligned}
$$

We now replace $u_{\alpha}^{1}(x, y)$ by its value $\sum_{i=1}^{N} \theta_{i, \alpha}(y) \frac{\partial u^{0}}{\partial x_{i}}(x)$ and obtain that the right-hand-side of this inequality is equal to

$$
-\int_{\Omega} \bar{D} \nabla u_{0}(x) \cdot \nabla u_{0}(x)+\sum_{\alpha=1}^{K} \int_{\Omega} f_{\alpha}(x) u_{0}(x) d x
$$

which is clearly zero because of the variational formulation of (61).

\section{$7 \quad$ Numerical results}

In this section we shall present some numerical results describing the asymptotical behaviour of the two-energy-group model $(4)(K=2)$. The goal is to test the accuracy of the homogenization procedure compared to a direct (expensive) approach. We have performed a simple one-dimensional simulation of an idealized reactor of length 1 , composed of $n$ identical cells. The periodicity cell, has a structure as sketched in figure 1. In a nuclear context, material A

Figure 1: periodicity cell structure

would correspond to water, surrounding fuel rods B (typically, uranium). However such a choice of materials would not create large $\epsilon$-scale oscillations, and the graphical output would simply display its smooth macroscopical trend (in real nuclear reactors large small-scale fluctuations are observed but with a more complicated pattern than that of figure 1 ; for example with mixed uranium 


\begin{tabular}{|c|c|c|c|c|c|c|c|}
\hline Medium & $A_{1}$ & $A_{2}$ & $\Sigma_{11}$ & $\Sigma_{22}$ & $\Sigma_{21}$ & $\sigma_{11}$ & $\sigma_{12}$ \\
\hline $\mathrm{A}$ & 1.200 & 0.100 & 2.500 & 1.500 & 0.001 & 0.000 & 0.001 \\
\hline $\mathrm{B}$ & 1.370 & 0.400 & 0.100 & 0.070 & 1.060 & 2.070 & 0.160 \\
\hline
\end{tabular}

Table 1: Numerical values used for the simulation

oxydes assemblies). Therefore, we chose to present a numerical simulation with fancy materials such that the two regions $\mathrm{A}$ and $\mathrm{B}$ correspond to high contrast materials. The numerical values of the different coefficients are presented in table 1.

In the two-energy-group model (4) three quantities are of interest. The first eigenvalue $\mu^{\epsilon}$, and its corresponding normalized eigenvector $\left(\phi_{1}^{\epsilon}, \phi_{2}^{\epsilon}\right)$, where $\phi_{1}^{\epsilon}$ is the fast neutrons flux and $\phi_{2}^{\epsilon}$ is the slow (or thermal) neutrons flux. We first computed directly these solutions (which, for small $\epsilon$, is an expensive task), and then compared them with the reconstructed homogenized solutions.

All computations are done with piecewise linear finite elements. In practice, we discretize the coefficients on a cell with $n_{\text {cell }}=50$ degrees of freedom, and then construct the domain as a juxtaposition of $N$ discretized cells. Using a power method, we compute the first eigenvalue and eigenvector of the direct problem with Dirichlet boundary conditions for $\epsilon=N^{-1}$ (corresponding to $N \times n_{\text {cell }}-1$ degrees of freedom). Alternatively, we compute the first eigenvalue $\mu^{\infty}$ and eigenvector $\left(\psi_{1}, \psi_{2}\right)$ on the discretized cell with periodic boundary conditions (the so-called infinite medium problem), and also the adjoint first eigenvector $\left(\psi_{1}^{*}, \psi_{2}^{*}\right)$ and the correctors $\left(\zeta_{1}, \zeta_{2}\right)$ that allow to compute the homogenized coefficients. Since the the homogenized problem has constant coefficients, we know its exact first eigenvalue and eigenvector (a sine function). Then we re-construct the fluxes by the following homogenized approximation

$$
\left\{\begin{array}{l}
\phi_{1}^{H, \epsilon}=\sin (x) \psi_{1}\left(\frac{x}{\epsilon}\right)+\epsilon \cos (x) \zeta_{1}\left(\frac{x}{\epsilon}\right) \\
\phi_{2}^{H, \epsilon}=\sin (x) \psi_{2}\left(\frac{x}{\epsilon}\right)+\epsilon \cos (x) \zeta_{2}\left(\frac{x}{\epsilon}\right) \\
\mu^{H, \epsilon}=\mu^{\infty}+\epsilon^{2} \frac{\pi^{2} \bar{D}}{\bar{\sigma}}
\end{array}\right.
$$

The constants $\bar{D}$ and $\bar{\sigma}$ are given by formulas (21) and (20), whereas corrector $\zeta$ is given by equation (22).

In table 2 are displayed the reference eigenvalue $\mu_{\epsilon}$ and its reconstructed counterpart $\mu^{H, \epsilon}$ for various number of cells. The last column shows the absolute error between the two, in p.c.m. unit (one p.c.m. is $10^{-5}$ ). The numerical estimate of the rate of convergence is

$$
v=\frac{\ln \left(\frac{e_{30}}{e_{10}}\right)}{\ln \left(\frac{10}{30}\right)} \simeq 3.20,
$$




\begin{tabular}{|c|c|c|r|}
\hline Number of cells & Reference $\mu^{\epsilon}$ & Reconstructed $\mu^{H, \epsilon}$ & error $\left(\times 10^{-5}\right)$ \\
\hline 5 & 2.29630 & 2.24501 & 5129 \\
\hline 10 & 1.75285 & 1.74068 & 1217 \\
\hline 15 & 1.65208 & 1.64729 & 479 \\
\hline 20 & 1.61681 & 1.61460 & 221 \\
\hline 25 & 1.60048 & 1.59947 & 101 \\
\hline 30 & 1.59161 & 1.59125 & 36 \\
\hline$\infty$ & 1.57257 & \multicolumn{2}{|c}{} \\
\hline
\end{tabular}

Table 2: Reference and reconstructed first eigenvalue for a high contrast cell

as expected from Theorem 3.2. This numerical estimate $\mu^{\epsilon}-\mu^{H, \epsilon}=\mathcal{O}\left(\epsilon^{3}\right)$ is of course much better than the convergence rate of $\mu^{\epsilon}$ to its limit $\mu^{\infty}$. In the one-energy group case, Malige [23] (building upon results in [29]) proved that the third-order term of the asymptotic expansion of $\mu^{\epsilon}$ is indeed zero if the periodicity cell is symmetric. We do not know if such a result would hold in the two-energy-group case. Our numerical results suggest at least that, if it were the case, the next non-zero term in the asymptotic expansion is larger than $\epsilon^{4}$.

In figure 2 are plotted the exact fast neutron flux $\phi_{1}^{\epsilon}$ and the reconstructed flux $\phi_{1}^{H, \epsilon}$ with the same normalization. In figure 3 are plotted the corresponding thermal fluxes $\phi_{2}^{\epsilon}$ and $\phi_{2}^{H, \epsilon}$. In our example, the addition of correctors

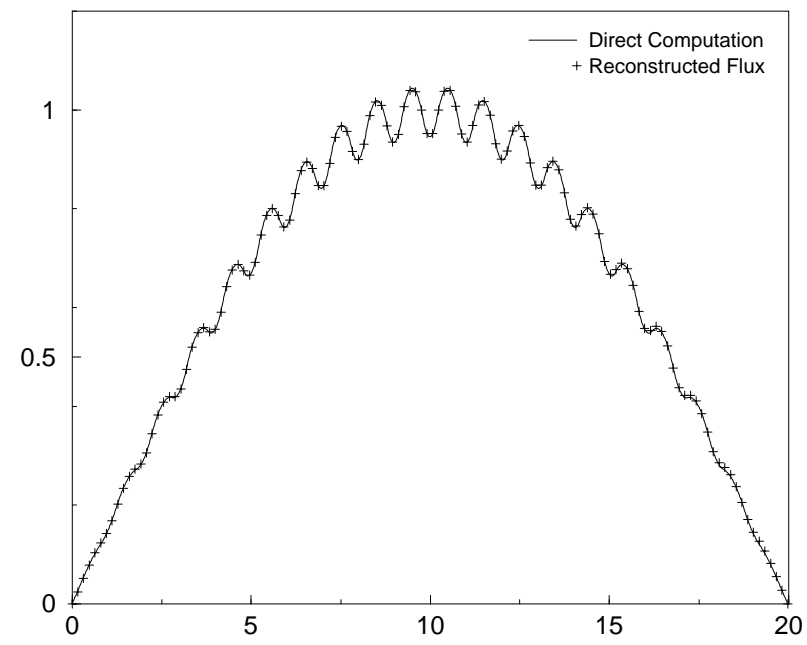

Figure 2: Fast neutrons flux, directly computed and reconstructed for 20 periodicity cells

$\epsilon \cos (x) \zeta_{\alpha}\left(\frac{x}{\epsilon}\right)$ does not improve significantly the reconstructed flux. However, 


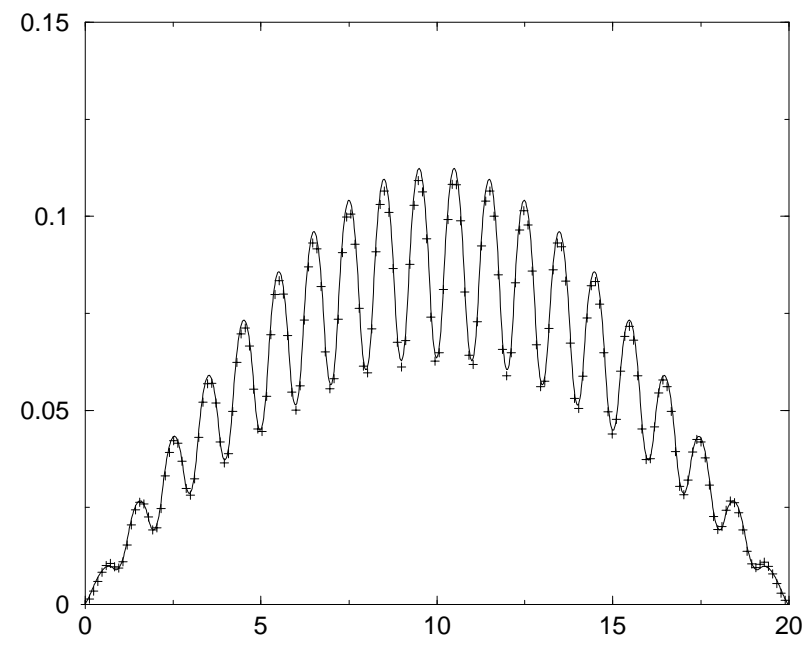

Figure 3: Thermal neutrons flux, directly computed and reconstructed for 20 periodicity cells

the correctors $\theta_{\alpha}=\zeta_{\alpha} / \psi_{\alpha}$ play a fundamental role in the computation of the homogenized diffusion coefficient. Indirectly, their influence on the homogenized eigenvalue is, here, about 1 percent, and grows rapidly if the contrast between the two media is increased.

\section{References}

[1] Altaire, G. Homogenization and two-scale convergence. SIAM J. Math. Anal. 23, 6 (Nov. 1992), 1482-1518.

[2] Allaire, G., and Bal, G. Homogénéisation d'une équation spectrale du transport neutronique. C. R. Acad. Sci. Paris Série I, t. 325 (1997), $1043-1048$.

[3] Allaire, G., And BaL, G. Homogenization of the criticality spectral equation in neutron transport. M2AN submitted (1998).

[4] Allaire, G., and Malige, F. Analyse asymptotique spectrale d'un problème de diffusion neutronique. C. R. Acad. Sci. Paris Série I, t.324 (1997), 939-944.

[5] Amann, H. Fixed point equations and nonlinear eigenvalue problems in ordered banach spaces. SIAM Review 18,4 (october 1976), 620-710.

[6] Anselone, P. Collectively Compact Operator Approximation Theory and Applications to Integral Equations. Prentice-Hall, Englewood Cliffs, N.J., 1971. 
[7] BAL, G. Couplage d'équations et homogénéisation en transport neutronique. Thèse de Doctorat de l'Université paris 6, 1997.

[8] BAL, G. First-order corrector for the homogenization of the criticality eigenvalue problem in the even parity formulation of the neutron transport. submitted to SIAM J. Appl. Math. (1998).

[9] BaL, G., AND Warin, X. An asymptotic analysis for the homogenization of p.w.r. assemblies - the transport case -. submitted to Nuclear Science and Engineering (1998).

[10] Benoist, P. Théorie du coefficient de diffusion des neutrons dans un réseau comportant des cavités. Note CEA-R 2278, 1964.

[11] Bensoussan, A., Lions, J.-L., and Papanicolaou, G. Asymptotic analysis for periodic structures. North-Holland, 1978.

[12] Bussac, J., and Reuss, P. Traité de neutronique. Hermann, Paris, 1978.

[13] Capdebosco, Y. Homogenization of a diffusion equation with drift. $C$. R. Acad. Sci. Paris Série I, t.327 9 (1998), 807-812.

[14] Chatelin, F. Spectral Approximation of Linear Operators. Computer Science and Applied Mathematics. Academic Press, 1983.

[15] Deniz, V. The theory of neutron leakage in reactor lattices. In Handbook of nuclear reactor calculations, Y. Ronen, Ed., vol. II. CRC Press, 1986, pp. $409-508$.

[16] Dorning, J.-J., Zhang, H., and Uddin, R. Systematic homogenization and self-consistent flux and pin power reconstruction for nodal diffusion - $\mathrm{i}$ : Diffusion equation-based theory. Nucl. Sci. Eng. 121 (1995), 226-244.

[17] Gilbarg, D., and Trudinger, N. Elliptic Partial Differential Equations of Second Order, 2nd ed. Comprehensive Studies in Mathematics. SpringerVerlag, 1983.

[18] Habetler, G., and Martino, M. The multigroup diffusion equations of reactor physics. rapport KAPL 1886, Knolls Atomic Power Laboratory, 1958 .

[19] Hornung, U., Ed. Homogenization and Porous Media, vol. 6 of Interdisciplinary Applied Mathematics. Springer, 1997.

[20] Kesavan, S. Homogenization of elliptic eigenvalue problems part 1 and 2. Appl. Math. Optim. 5 (1979), 153-167,197-216.

[21] Krein, M., and Rutman, M. Linear operators leaving invariant a cone in a banach space. Publication AMS, Functional Analysis and Measure Theory 10 (1962), 199-325. 
[22] Larsen, E., and Hughes, R. Homogenized diffusion approximations to the neutron transport equation. Nucl. Sci. Eng. 79 (1980), 274-280.

[23] Malige, F. Etude mathématique et numérique de l'homogénéisation des assemblages combustibles d'un cœur de réacteur nucléaire. Thèse de Doctorat de l'École Polytechnique, 1996.

[24] Malige, F., and Vaudescal, J.-L. An asymptotic analysis for the systematic homogenization of pwr assemblies. the diffusion case. Tech. Rep. HI-72/97/008/0, EDF-DER, 1997.

[25] Murat, F., and Tartar, L. H-convergence. Séminaire d'Analyse Fonctionnelle et Numérique de l'Université d'Alger, mimeographed notes (78). English translation in Topics in the mathematical modelling of composite materials, Cherkaev A., Kohn R.V., Editors, Progress in Nonlinear Differential Equations and their Applications, 31, Birkhaüser, Boston (1997).

[26] Nguetseng, G. A general convergence result for a functional related to the theory of homogenization. SIAM J. Math. Anal., 20 (1989), 608-623.

[27] Planchard, J. The problem of the power distribution inside the fuel assemblies of pwr reactors. E.D.F. Bulletin de la direction des Etudes et Recherches série C, 4 (1989), 1-17.

[28] Planchard, J. Méthodes mathématiques en neutronique. In Collection de la Direction des Études et Recherches d'EDF. Eyrolles, Paris, 1995.

[29] Santosa, F., and Vogelius, M. First-order corrections to the homogenized eigenvalues of a periodic composite medium. SIAM J. Appl. Math. 53 (1993), 1636-1668.

[30] Stampacchia, G. Le problème de dirichlet pour les équations élliptiques du second ordre à coefficients discontinus. Ann. Inst. Fourier 15,1 (1965), $189-258$. 